\title{
Air-chemistry "turbulence": power-law scaling and statistical regularity
}

\author{
H.-m. Hsu ${ }^{1}$, C.-Y. Lin ${ }^{2}$, A. Guenther ${ }^{1}$, J. J. Tribbia ${ }^{1}$, and S. C. Liu ${ }^{2}$ \\ ${ }^{1}$ National Center for Atmospheric Research, Boulder, Colorado, USA \\ ${ }^{2}$ Research Center for Environmental Changes, Academia Sinica, Taipei, Taiwan \\ Received: 17 December 2010 - Published in Atmos. Chem. Phys. Discuss.: 22 March 2011 \\ Revised: 1 July 2011 - Accepted: 22 July 2011 - Published: 18 August 2011
}

\begin{abstract}
With the intent to gain further knowledge on the spectral structures and statistical regularities of surface atmospheric chemistry, the chemical gases $\left(\mathrm{NO}, \mathrm{NO}_{2}, \mathrm{NO}_{\mathrm{x}}, \mathrm{CO}\right.$, $\mathrm{SO}_{2}$, and $\left.\mathrm{O}_{3}\right)$ and aerosol $\left(\mathrm{PM}_{10}\right)$ measured at 74 air quality monitoring stations over the island of Taiwan are analyzed for the year of 2004 at hourly resolution. They represent a range of surface air quality with a mixed combination of geographic settings, and include urban/rural, coastal/inland, plain/hill, and industrial/agricultural locations. In addition to the well-known semi-diurnal and diurnal oscillations, weekly, and intermediate ( $20 \sim 30$ days) peaks are also identified with the continuous wavelet transform (CWT). The spectra indicate power-law scaling regions for the frequencies higher than the diurnal and those lower than the diurnal with the average exponents of $-5 / 3$ and -1 , respectively. These dual-exponents are corroborated with those with the detrended fluctuation analysis in the corresponding time-lag regions. These exponents are mostly independent of the averages and standard deviations of time series measured at various geographic settings, i.e., the spatial inhomogeneities. In other words, they possess dominant universal structures. After spectral coefficients from the CWT decomposition are grouped according to the spectral bands, and inverted separately, the PDFs of the reconstructed time series for the highfrequency band demonstrate the interesting statistical regularity, -3 power-law scaling for the heavy tails, consistently. Such spectral peaks, dual-exponent structures, and powerlaw scaling in heavy tails are important structural information, but their relations to turbulence and mesoscale variability require further investigations. This could lead to a better understanding of the processes controlling air quality.
\end{abstract}

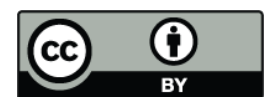

Correspondence to: H.-m. Hsu (hsu@ucar.edu)

\section{Introduction}

During the last decades, a large quantity of near-surface air quality measurements, including aerosol and chemical gases, has been accumulated. Significant progress in understanding chemical processes/reactions and their interactions with meteorological processes in the lower atmosphere have been made, and some of them are described in Seinfeld and Pandis (2006). However, there is still a lack of information on how chemical species are related to various dynamical scales in both time and space. Of course, the basic diurnal and semidiurnal oscillations found in the chemical observations (e.g., Hastie et al., 1993; Güsten et al., 1998; Mao and Talbot, 2004, Talbot et al., 2005; Stephens et al., 2008) are caused by variations in photochemical reactivity and traffic emissions, respectively, in addition to the diurnal boundary-layer variability. Stephens et al. (2008) identified weekly signals in their study of the atmospheric chemical constituents in Mexico City, most likely due to the lower weekend emissions of ozone $\left(\mathrm{O}_{3}\right)$ precursors including nitrogen oxides $\left(\mathrm{NO}_{\mathrm{x}}\right.$, i.e., $\mathrm{NO}$ and $\mathrm{NO}_{2}$ ) and reactive volatile organic compounds (VOCs), and reported references on this subject for other major cities and metropolitan regions. Is our fundamental knowledge from observed time series of different species only limited to these famous and obvious oscillations? Further investigations of observed atmospheric chemical data in terms of dynamic scales are extremely important in gaining a fundamental knowledge for linking atmospheric chemistry and dynamics. In this paper we introduce the spectral and statistical analysis methodology to comprehensively treat the observed time series and reveal their spectral structures and statistical regularities.

It is well known that one of the fundamental representations of atmospheric motions is their spectral structures. On one hand, distinct peaks indicate the dominant scales. On the other hand, there may be "scale-free" in a spectral

Published by Copernicus Publications on behalf of the European Geosciences Union. 
range, which suggests the scale invariance. Such information is important in identifying the gross structure of the atmospheric motions considered in terms of scales. Kolmogorov (1941) expressed such scale invariance in turbulence as the $-5 / 3$ power-law scaling. Such scaling was also found persistently in larger scales (e.g., Nastrom and Gage, 1985; Gage and Nastrom, 1986; Lindborg, 1999; and others). Gage (2004) gave a thorough review on the mesoscale variability of $-5 / 3$ scaling, and Tuck (2008) summarized the atmospheric turbulence including chemistry in the upper atmosphere. Because of Kolmogorov's establishment of the power-law scaling of turbulence in the inertial subrange, the modern large-eddy simulation methodology is thus based and developed on such foundation. For the atmospheric chemistry near the surface, it is necessary to analyze the available data from various measurements to reveal their spectral and statistical structures.

From the modeling point of view, numerical simulations/forecasts of chemical weather/climate are computationally heavy at very high resolutions, owing to the number of the predictive equations for reactive chemical species being included in the calculations (Emmons et al., 2010; Fast et al., 2005; Grell et al., 2005). While the numerical methods to solve the partial differential equations employed in numerical models have advanced tremendously (e.g., Miller and Smolarkiewicz, 2008), the largest uncertainty in simulations/forecasts is contained in various parameterizations for the unresolved processes in numerical models because of the limitations of resolution. For the traditional weather/climate models at different resolutions, various degrees of necessary parameterizations are complex enough. The uncertainty in chemical reactions and emissions further complicates the parameterization issues, in addition to the interactions between chemical reactions (fast/slow) and meteorological processes. The spectral and statistical structures obtained from the observed data can be effectively used to formulate and/or constrain possible parameterizations for the unresolved chemi$\mathrm{cal} /$ physical processes for future numerical simulations and forecasts of chemical weather/climate. They can further suggest possible fine-resolution measurements necessary to consider the chemical processes involving short-lived species.

In order to evaluate the numerical results from model simulations/forecasts, objective assessments using various statistical tools are well established (e.g., Wilks, 1995; Jolliffe and Stephenson, 2003), but the error information is static with simple statistical measures such as correlation coefficient, root mean square error, and others. Comparisons between the observed and the modeled in terms of their spectral components can be very helpful to understand the limit of the accuracy of the model results, across-scale. In other words, objective error analysis using spectral decompositions can assess the accuracy in terms of dynamic scales. A simple example is that the model resolution is fine enough inside the nonhydrostatic regime, but the high correlations between the observed and the modeled only appear in the hy- drostatic regime. The spectral structures of the observed data are critical for an objective assessment of model results.

Here, both spectra from the continuous wavelet transform (CWT) and the detrended fluctuation analysis (DFA) for the hourly measurements of chemical species and aerosol at 74 air quality monitoring stations spread over the island of Taiwan during 2004, are presented to introduce our analysis methodology and to illustrate the results. In addition to the various oscillatory peaks, two power-law scaling regions of the high-frequency (higher than the diurnal) and low-frequency (lower than the diurnal) bands have the average CWT exponents of $-5 / 3$ and -1 , respectively. These exponents are corroborated with the power-law exponents from the DFA in the corresponding time-lag bands. The highfrequency exponent of $-5 / 3$ is not only coincide with the Kolmogorov's $-5 / 3$ turbulence spectral exponent, but also implies its possible relationship with the local smaller-scale turbulence.

Moreover, the reconstructed signals from the decomposed CWT coefficients in the high-frequency band demonstrate the statistical regularity in the high-intensity range: The heavy tail in the PDFs has a power-law scaling of -3 . These results are valuable and useful, as our initial attempt to understand the spectral structures and statistical regularity of the measured chemical data. As far as we know this methodology has never been applied to surface chemical data.

This paper is organized as follows. The data and analysis methodology are described in Sect. 2. The results from trace gases and aerosol are discussed in Sects. 3 and 4, respectively. Summary and concluding remarks are given in Sect. 5.

\section{Data and analysis method}

To understand the complex atmospheric processes in evaluating air quality, continuous efforts are being made in many regions to provide more high-frequency and high-quality data by increasing the number of measurement sites and installing more accurate instruments. Over the island of Taiwan, the concern over air quality impacts has resulted in the development of a network of continuous measurements of important trace gases and aerosol since 1970s.

All the data were collected at the air monitoring stations spread over the island in urban/rural, coastal/inland, plain/hill, and industrial/agricultural areas, but clustered over two major metropolitans, Taipei and Kaohsiung. Figure 1 depicts the locations where measurements were made. A total of 74 stations provided complete hourly time series during 2004, and the data consist of chemical species of $\mathrm{CO}, \mathrm{SO}_{2}$, $\mathrm{O}_{3}, \mathrm{NO}, \mathrm{NO}_{2}, \mathrm{NO}_{\mathrm{x}}$, and aerosol $\left(\mathrm{PM}_{10}\right)$. Data precision is always a concern on the back of analyst's mind, no matter how careful the measurements were made. No measured data are perfect. Then the question becomes how much tolerance we can afford for the data analysis. Of course, one needs 


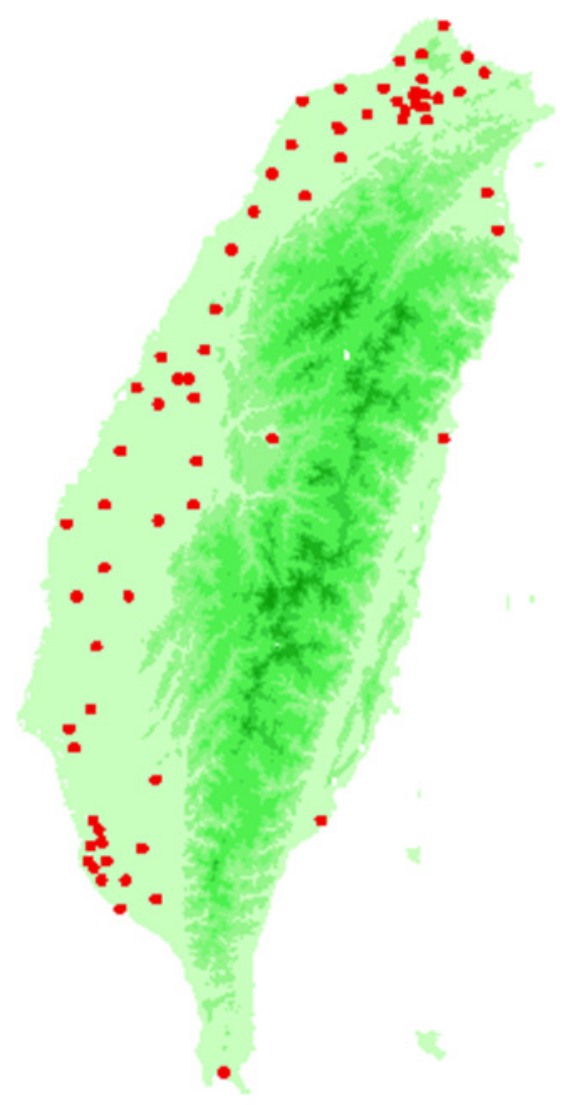

Fig. 1. The island of Taiwan with the locations of 74 air quality monitoring stations. The light and dark green indicate the coastal plain and mountain peaks.

to device various reasonable procedures to quality-control the raw data. Should the data are claimed to be qualitycontrolled from the data sources, one still can device/employ data-analysis methods to see if the data sets contain physical/dynamic sense. Here we employee two independent spectral methods as described in following paragraphs. Furthermore, all the time series were cut into half, and the decomposed separated. The corresponding spectra are almost identical except that the exponents from the halved time series cannot reach those from the original ones in the lowfrequency end of the spectra.

For all data, the average of each time series is removed from all the time series. Then they are normalized by their own respective standard deviations. We are primarily interested in learning various statistical structures in the data. The resulting time series are first decomposed by the continuous wavelet transform (CWT) into the time-frequency domain. The global spectra are obtained by averaging the timefrequency spectra. Even though the conventional Fourier transform can perform similarly, the resulting spectra are much noisier due to the non-periodic, nonlinear and/or nonstationary nature of the data.
Regarding to the choice of basis functions for the wavelets, the chemical species have clear diurnal and semi-diurnal cycles in the time domain, so a localized wave-like basis function is appropriate. This allows distinct temporal signals in the time series to be quantified. Among other continuous wavelet functions such as the Paul and Mexican-hat functions, the Morlet wavelet, a plane sinusoidal wave modulated by a Gaussian envelope (Morlet et al., 1982) is the most suitable for oscillatory signals. As in other transform techniques, problems can develop at the ends of the finite-length time series, due to non-periodic nature of the data. Necessary steps such as padding zeros at the end of the time series mitigate such problems. Our approach follows those of Torrence and Compo (1998), and Kestin et al. (1998). Additional details and applications of the CWT can be found in Farge (1992), Mallat (1999), Meyers et al. (1993), and Lau and Weng (1995).

Primarily to corroborate our results from the continuous wavelet transform, another analysis method is considered here. The detrended fluctuation analysis (DFA), which was originally developed to investigate the highly irregular behavior of DNA and other biological data (Peng, et al., 1992, 1994, 1995), is adopted to analyze the hourly measured data from air monitoring stations. Although the DNA and other biological data normally fluctuate in a complex, apparently erratic manner, DFA was able to uncover the underlying long-range power-law correlations reminiscent of physical systems near a critical point.

The detrended fluctuation is calculated as an additional step to the regular fluctuation. The regular fluctuation is defined in terms of the difference between the average of the square and the square of the average on a difference of two elements of a walk series. The interval between two elements in the calculation varies systematically in a sequential manner. The walk series is defined as the running sum of any series. The additional step is to subtract the best fit from the two walk elements in that interval (the trend). The regular fluctuation is known to have spurious crossover behavior, while the detrended one shows linear behavior on the log$\log$ plot for all length scales up to the data size (Peng, et al., 1994). They applied DFA to clearly confirm the difference between coding and non-coding sequences in a DNA walk based on the argument of long-range power-law correlations implying scale invariance of DNA, and showed that the former are less correlated than the latter for the sequence length scale which is close to the characteristic DNA patch size in the coding region. Here, we use the same procedure to our observed chemical data.

There is a special algebraic relationship between the exponents of CWT and DFA. Originally, such relationship was found in the long-range correlation properties of coding and noncoding DNA sequences using the Fourier transform and DFA (Peng et al., 1993; Buldyrev et al., 1995; Hausdorff et al., 1996; Heneghan and McDarby, 2000). Let the power spectra, $S(f) \sim f^{-\beta}$, where $\beta$ is the power spectra exponent 
and $f$ the frequency, and the DFA spectra, $F(l) \sim l^{\alpha}$, where $\alpha$ is the DFA exponent and $l$ the time lag. The relationship is $\beta=2 \alpha-1$, and is evaluated by decomposing the observed chemical data.

Power-law spectra provide basic information on intensity at specific frequencies. Generally, power-law spectra measure characteristics of distinct peaks and/or straight powerlaw scaling, as revealed in Hsu et al. (2006). For example, the exponent $(\beta)$ of the power-law scaling can be classified into distinct ranges (Wornell, 1996): $1<\beta<3$ represents non-stationary random processes of finite power in any finite data interval (fractional Brownian motion); $-1<\beta<1$ represents stationary Gaussian processes corresponding to the derivative of a fractional Brownian motion (fractional Gaussian noise). The special cases are the classical Brownian motion $(\beta=2)$ and the stationary white Gaussian noise $(\beta=0)$. For exponents steeper than -3 and gentler than 1 , so far, no explanatory theory exists. For DFA, $1<\alpha<2$ is for the non-stationary and $0<\alpha<1$ for the stationary. Our present analyses differentiate the chemical data into stationary/nonstationary processes based on the frequency bands.

All the chemical data are decomposed into spectral coefficients for each frequency, and coefficients are inverted back to the physical spaces according to their characteristic spectral bands, using CWT. Statistical measures from the reconstructed time series are compared. Because the time series are normalized (zero mean and unit variance), we focus on their skewness and kurtosis to characterize the reconstructed for different frequency bands.

\section{Chemical gas spectra and PDF}

Trace gases (NO, $\mathrm{NO}_{2}, \mathrm{NO}_{\mathrm{x}}, \mathrm{CO}, \mathrm{SO}_{2}$, and $\mathrm{O}_{3}$ ) measured at the air quality monitoring stations possess various properties near the surface. In addition to the influences of meteorological variability, local emissions and chemical reactions play important and different roles for their presence and lifetimes in the lower atmosphere.

Nitrogen oxides $\left(\mathrm{NO}_{\mathrm{x}}\right.$, i.e., $\mathrm{NO}$ and $\left.\mathrm{NO}_{2}\right)$ have a central regulatory role in the free radical and oxidizing photochemistry of the troposphere. This photochemistry regulates the lifetime of methane and the production of tropospheric ozone and secondary aerosol, all of which have important roles in the natural and anthropogenic greenhouse effect. The ozone produced in the atmosphere as a result of these nitrogen oxides can affect vegetation growth and human health. Sources of $\mathrm{NO}_{\mathrm{x}}$ include fertilizer use, fossil fuel combustion, biomass burning, lightning and soil (IPCC, 2007). The dominant sink of $\mathrm{NO}_{\mathrm{x}}$ in the atmosphere is its conversion into nitric acid $\left(\mathrm{HNO}_{3}\right)$ and peroxyacetylnitrate (PAN), which are eventually removed by dry or wet deposition. Anthropogenic emission of $\mathrm{NO}_{\mathrm{x}}$ is currently one of the major causes of acid deposition, which can directly remove $\mathrm{NO}_{\mathrm{x}}$ from the atmosphere and degrade water quality.
$\mathrm{CO}$ has a relatively long atmospheric lifetime, ranging from 10 days in summer in the tropics to more than a year over polar areas in winter. Sources of atmospheric CO include fossil fuel combustion and biomass burning, along with the oxidation of natural and anthropogenic methane and nonmethane hydrocarbons (NMHC). Major sinks include reactions with $\mathrm{OH}$ and surface deposition. $\mathrm{CO}$ influences the oxidation capacity of the atmosphere through interaction with hydroxyl radicals $(\mathrm{OH})$, which are a major sink for methane and halocarbons.

Sulphur dioxide $\left(\mathrm{SO}_{2}\right)$ is oxidized by hydroxyl radicals $(\mathrm{OH})$ to sulphuric acid, which then forms aerosols through photochemical gas-to-particle conversion. While $\mathrm{SO}_{2}$ reacts much more slowly with $\mathrm{OH}$ than does $\mathrm{NO}_{2}, \mathrm{SO}_{2}$ dissolves readily in suspended liquid droplets in the atmosphere. Sources of $\mathrm{SO}_{2}$ include fossil fuel combustion by industry, biomass burning, volcanic release and the oxidation of biogenic dimethylsulphide (DMS) from the oceans (IPCC, 2001). Major $\mathrm{SO}_{2}$ sinks are oxidation by $\mathrm{OH}$ and deposition onto wet surfaces. Anthropogenic $\mathrm{SO}_{2}$ has been a major source of acid deposition throughout the industrial era. The mole fractions of $\mathrm{SO}_{2}$ show large variations in both space and time because of its short lifetime and uneven anthropogenic source distribution.

Ozone $\left(\mathrm{O}_{3}\right)$ in the troposphere plays an important role in the atmospheric environment through radiative and chemical processes. Tropospheric $\mathrm{O}_{3}$ originates from downward mixing from the stratosphere and in-situ photochemical production in the presence of nitrogen oxides and volatile organic compounds. $\mathrm{O}_{3}$ is destroyed by various processes including chemical reactions with $\mathrm{NO}$, the hydroperoxy radical $\left(\mathrm{HO}_{2}\right)$ and $\mathrm{OH}$, and deposition at the Earth's surface. The lifetime of tropospheric ozone varies from one or a few days in the boundary layer to a few tens of days or even a few months in the free troposphere.

Although these chemical gases have very different source and sink and the chemical reactions can be fast/slow, they have some very similar spectral and statistical characteristics as illustrated in the following analyses.

\subsection{Autocorrelation}

To identify the statistical nature of any time series, the autocorrelation is the first analysis to perform, particularly for apparently random-looking data. If the autocorrelation is identical to zero, then the series is uncorrelated. Gaussian white noise is an example. Otherwise, additional analyses are required. Our intuition is that autocorrelations should decay exponentially such that a de-correlation temporal scale may be defined. When the decay is slower than the exponential but faster than the linear, the possibility could be power-law. Thus no length scale can be defined which is sometime called scale-free.

Figure 2 illustrates the autocorrelations of $\mathrm{NO}, \mathrm{NO}_{2}, \mathrm{NO}_{\mathrm{x}}$, $\mathrm{CO}, \mathrm{SO}_{2}$, and $\mathrm{O}_{3}$ time series at 74 stations for 2004. In each 

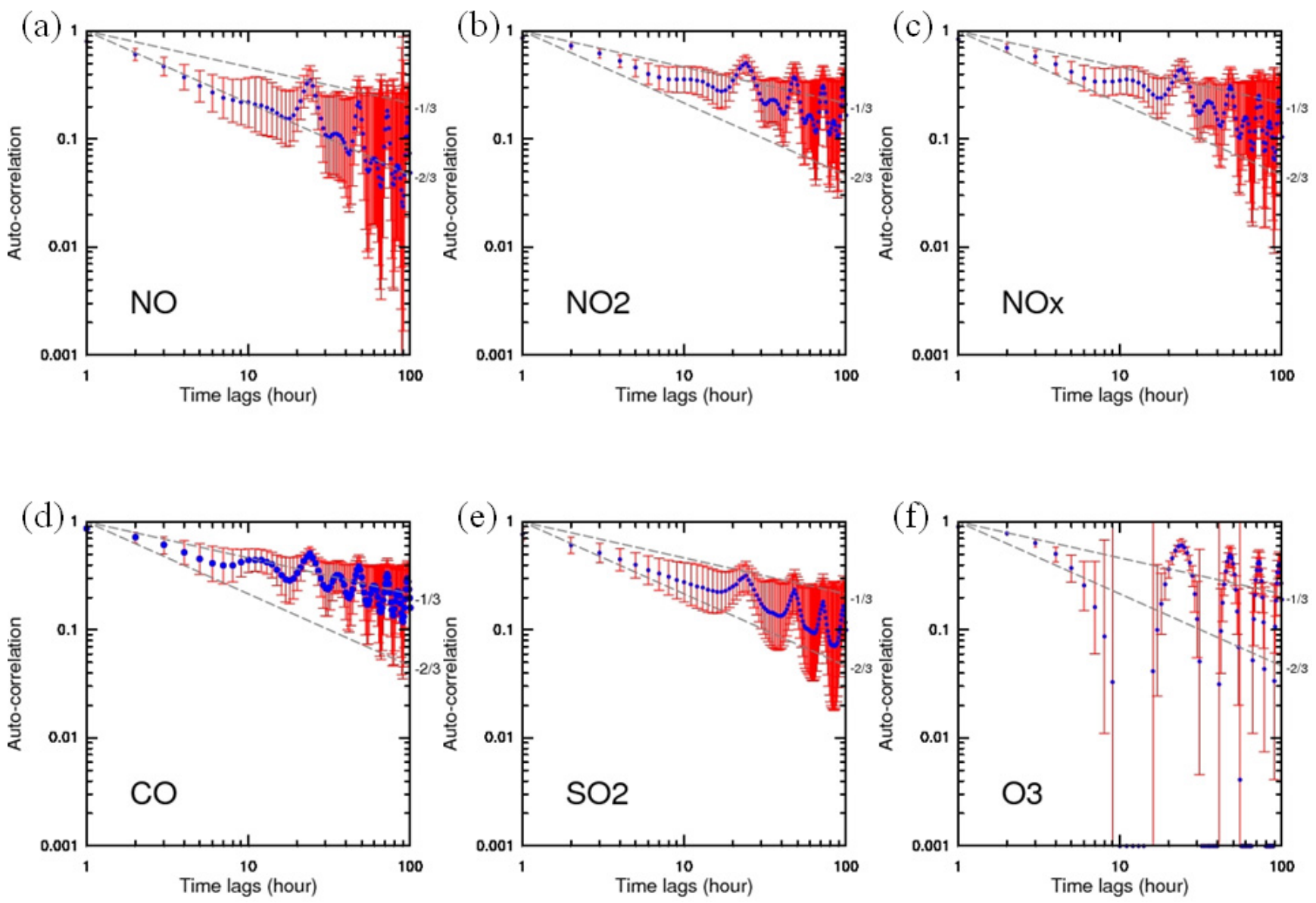

Fig. 2. The auto-correlations (in log-log scale) of the gases, $\mathrm{NO}$ (panel a), $\mathrm{NO}_{2}$ (panel b), $\mathrm{NO}_{\mathrm{x}}($ panel $\mathbf{c}$ ), $\mathrm{CO}$ (panel d), $\mathrm{SO} 2$ (panel e), and $\mathrm{O}_{3}$ (panel $\mathbf{f}$ ). The blue dots are the 74-station averages, and the red bars are standard deviations from the averages. The grey dash lines indicate the power-law scaling of $-1 / 3$ and $-2 / 3$.

panel, the ensemble-averages (the blue dots) of the autocorrelation coefficients over the stations are plotted in the log-log scale, and their standard deviations are in red. At large time lags, oscillations are apparent in all chemical species and are identified later in the power spectra. However, power-law scaling of the autocorrelation coefficients with different exponents exists at different small time lags. They all are scalefree. Not only do the exponents vary between $-1 / 3$ and $-2 / 3$ for $\mathrm{NO}, \mathrm{NO}_{2}, \mathrm{NO}_{\mathrm{x}}, \mathrm{CO}, \mathrm{SO}_{2}$, and $\mathrm{O}_{3}$, but also the standard deviations increase as the lags become large. The increases of standard deviations indicate the power-law scaling of the autocorrelation coefficients is less consistent among stations. Additionally, among different species the lags for the power-law scaling extend between 6 to $18 \mathrm{~h}$, except for only $3 \sim 4 \mathrm{~h}$ for $\mathrm{O}_{3}$. All of them reach the first 24 -hour high after the power-law declines. The shorter lags of the powerlaw scaling involve the strength of the semi-diurnal oscillations, which involve the emissions of pollutants during rush hours.
The ozone autocorrelation coefficients (Fig. 2f) exhibit very strong diurnal oscillations because its formation near the surface primarily depends on the photochemical reactions by the solar radiation. Also the relatively short lag of $\mathrm{O}_{3}$ coefficients indicates the volatile and quick reactive nature of $\mathrm{O}_{3}$ at the surface level with other species during its formation. Other species, $\mathrm{NO}, \mathrm{NO}_{2}, \mathrm{NO}_{\mathrm{x}}, \mathrm{CO}$, and $\mathrm{SO}_{2}$ are also reactive, but they are typically dominated by the surface emissions and are much less variable.

\subsection{Power spectra}

For the statistical characterizations of any variable, the classical measures of different orders, such as mean, variance, skewness and kurtosis, are very useful. However, to gain additional dynamical knowledge, the analysis of spectral decompositions can shed some light on the structures of the system in terms of dynamic scales. It is well known that the obvious advantage for spectral decompositions of data is to identify distinct spectral peaks for the dominant scales in the data. Moreover, some systems possess multiscale or 

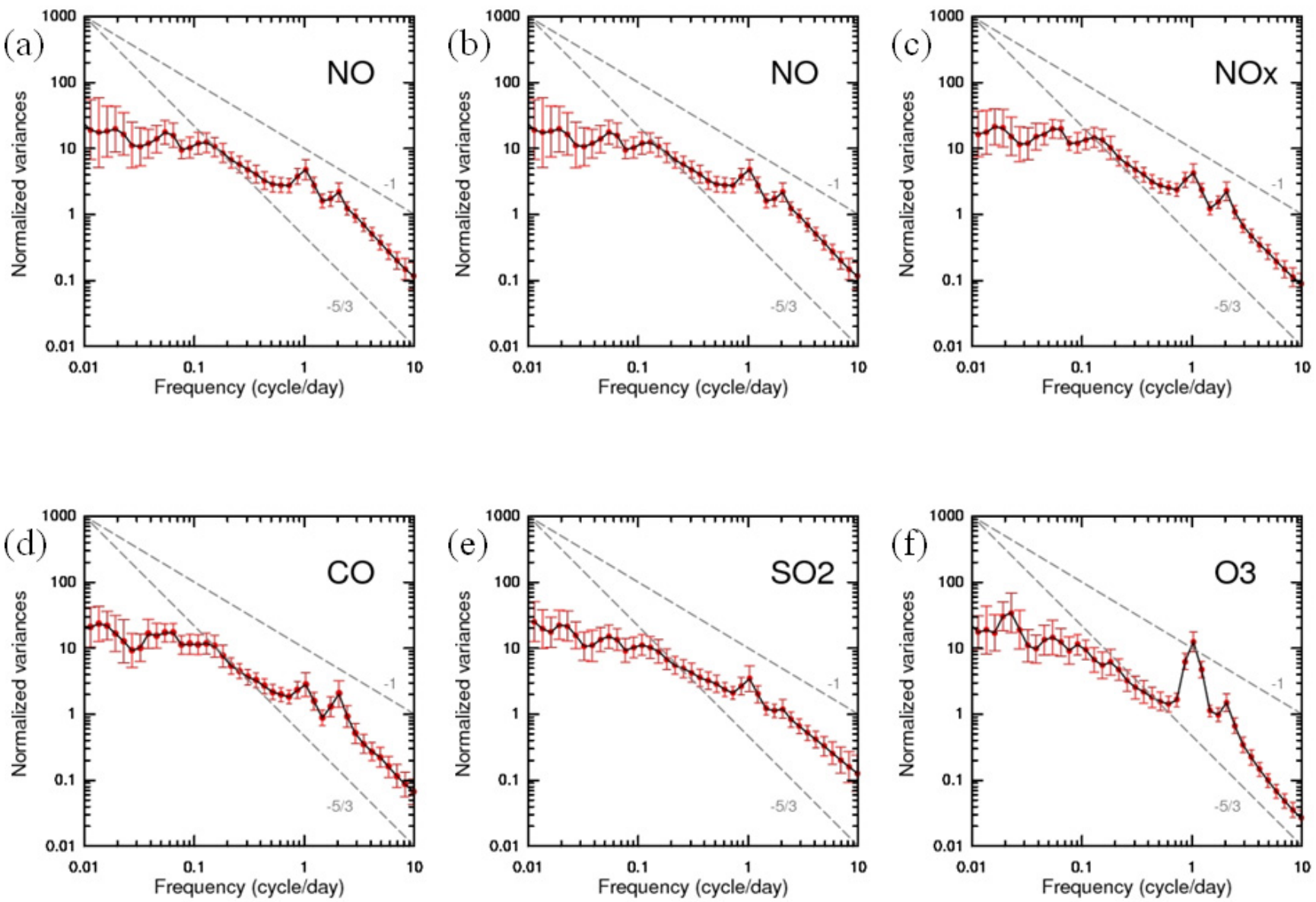

Fig. 3. The CWT spectra (in log-log scale) of the gases, $\mathrm{NO}$ (panel a), $\mathrm{NO}_{2}$ (panel b), $\mathrm{NO}_{\mathrm{x}}$ (panel c), $\mathrm{CO}$ (panel d), $\mathrm{SO} 2$ (panel e), and $\mathrm{O}_{3}$ (panel $\mathbf{f}$ ). The black curves are the 74-station averages, and the red bars are standard deviations from the averages. The grey dash lines indicate the power-law scaling of $-5 / 3$ and -1 .

even scale-free characteristics. All the six chemical species observed at the air monitoring stations are analyzed by applying CWT and DFA such that independent spectra are obtained. Then spectra of 74 stations are averaged to obtain the mean spectra for each chemical species and their standard deviations.

The global spectra from the continuous wavelet transform (CWT) are shown in Fig. 3. All of them have clear spectral peaks at semi-diurnal and diurnal frequencies, corresponding to the local rush-hour emissions in the mornings and evenings and the photochemical reactions variability, respectively, in addition to the diurnal planetary boundary layer evolutions driven primarily by the daytime heating and nighttime cooling at the surface (e.g., Hastie et al., 1993; Güsten et al., 1998; Mao and Talbot, 2004, Talbot et al., 2005; Stephens et al., 2008). Among $\mathrm{NO}, \mathrm{NO}_{2}, \mathrm{NO}_{\mathrm{x}}, \mathrm{CO}$, and $\mathrm{SO}_{2}$ spectra, these two peaks have about the same pattern, i.e., the diurnal one is stronger than the semi-diurnal one. However, the diurnal peak is an order of magnitude larger than the semi-diurnal one for the $\mathrm{O}_{3}$ spectrum, indicating the primary influence of solar radiation for $\mathrm{O}_{3}$ generation through the photochemical processes and consisting with large oscillations appearing its autocorrelation coefficients (Fig. 3f).

On the lower frequency band, there are at least 3 identifiable peaks. The weekly peak between 5 to 10 days is present for all the gases, except that ozone has two distinct peaks, 5 and 10 days. Stephens et al. (2008) showed this weekly peak was the weekend effect in Mexico City because of the lower emissions during weekends, and provided references for similar observations in a number of other major cities and metropolitan areas. On the other hand, Kim et al. (2010) recently argued for the importance of the weeklyscale synoptic features in the NCAR/NCEP reanalysis data (Kalnay et al., 1996). This weekly peak could also be synoptic on an annual base due to long-range transport. The synoptic features traversing the island of Taiwan primarily are the springtime Mei-yu fronts, the summertime tropical cyclones, and the wintertime fronts and anticyclones. Continental anticyclones associated with frontal systems usually form at a rate of about once a week (Bachmeier et al., 1996). Lin et 
al. $(2004,2005)$ provide evidence that the long-range transport of $\mathrm{CO}, \mathrm{SO}_{2}$ and $\mathrm{PM}_{10}$ to Taiwan following the cold front of winter monsoon that originates in the Asian continent.

The broad peak between 30 and 100 days is intraseasonal, and could be related to the tropical convective circulations such as the Madden-Julian oscillation (Madden and Julian, 1994). However, the upper bound of this broad peak is about 100 days. With the data size of 1 year, it is less confident to claim the reality of the intraseasonal peak. But, for the lower bound of 30 days, the periodic signal should be significant. The physical significance of the Madden-Julian Oscillation is well-known. Nonetheless, to confirm the broad spectral peak between 30 and 100 days using multi-year chemical data sets are required. Between the diurnal and the probable intraseasonal peaks, another peak exists between 20 and 30 days, the intermediate-scale. Since the analysis period is a full year and Taiwan is situated in the subtropical climate, the synoptic influence could come from the well-known mid-latitude features such as extratropical cyclones, and the Tropics is famous for the intraseasonal oscillations of tropical convection. It is plausible that over the island and its vicinity, the interactive influences between the mid- and low-latitude features from season to season could produce this broad intermediatescale peak.

Furthermore, there are power-law scaling with two average exponents for all the chemical species, about $-5 / 3$ and -1 for the high-frequency band (HFB) of frequencies higher than the diurnal and the low-frequency band (LFB) of frequencies lower than the diurnal, respectively (Table 1). The $-5 / 3$ scaling actually varies between $-5 / 2$ and -1 , and the -1 scaling varies between -1 and $-1 / 2$. In terms of powerlaw scaling, the dual-exponent structure appears in the spectra, and is separated by the diurnal and semi-diurnal peaks. The $-5 / 3$ exponent has been referred to as the mesoscale variability. The $-5 / 3$ power-law scaling of the kinetic-energy spectrum for homogeneous isotropic turbulence in the inertial sub-range is the most celebrated (Kolmogorov, 1941). Observations also indicate a $-5 / 3$ scaling at scales of tens and hundreds of kilometers (Lilly, 1983, 1989; Nastrom, et al., 1984; Nastrom and Gage, 1985; Cho et al., 1999a, b) in the kinetic and potential energy spectra. Gage (2004) gave a thorough review of the mesoscale variability of $-5 / 3$ scaling. Although the previous studies indicated that the kinetic and potential energy spectra have simple -3 and $-5 / 3$ for largeand meso-scales, respectively, our chemical spectra illustrate a mix of distinct peaks (semi-diurnal, diurnal, weekly, and intermediate) and power-law scaling $(-1$ and $-5 / 3)$ regions, representing environmental (meteorological) influences and the chemical reactions. The mesoscale variability of the $-5 / 3$ scaling may coincide to our high-frequency band exponent of $-5 / 3$, but the local smaller-scale turbulence may play an important role for the universal structure at high-frequency band.
Table 1. The ranges of CWT and DFA exponents for chemical gases and aerosol data measured at the 74 air quality monitoring stations.

\begin{tabular}{lrrrr}
\hline & $\begin{array}{r}\mathrm{CWT} \\
\text { High FB }\end{array}$ & $\begin{array}{r}\text { CWT } \\
\text { Low FB }\end{array}$ & $\begin{array}{r}\text { DFA } \\
\text { Short TLB }\end{array}$ & $\begin{array}{r}\text { DFA } \\
\text { Long TLB }\end{array}$ \\
\hline $\mathrm{NO}, \mathrm{NO}_{2}$, & $-1 \sim-5 / 2$ & $-1 / 2 \sim-1$ & $1 \sim 3 / 2$ & $3 / 4 \sim 1$ \\
$\mathrm{NO}_{\mathrm{x}}, \mathrm{CO}, \mathrm{SO}_{2}$ & & & & \\
\hline $\mathrm{O}_{3}$ & $-2 \sim-5 / 2$ & $-3 / 4 \sim-3 / 2$ & $5 / 4 \sim 7 / 4$ & $3 / 4 \sim 1$ \\
\hline $\mathrm{PM}_{10}$ & $-3 / 2 \sim-3$ & $-3 / 4 \sim-5 / 4$ & $1 \sim 3 / 2$ & $3 / 4 \sim 5 / 4$ \\
\hline
\end{tabular}

\subsubsection{DFA spectra}

As described earlier, the detrended fluctuation analysis (DFA) is superior to the original fluctuation analysis for identifying the power-law scaling and the long-range correlation. The DFA spectra from the $\mathrm{NO}, \mathrm{NO}_{2}, \mathrm{NO}_{\mathrm{x}}, \mathrm{CO}, \mathrm{SO}_{2}$, and $\mathrm{O}_{3}$ time series are displayed in Fig. 4. The detrended fluctuations are positively correlated to the time-lags, i.e., the time separations between any 2 times. It is apparent that the exponents are larger than 1 for the small time-lag band (STLB, the time lags smaller than the diurnal) and are smaller than 1 for the large time-lag band (LTLB, the time lags larger than the diurnal) for all of them. The transition of the exponents from those more than 1 to those less than 1 occurs about the diurnal time lag. Also the standard deviations from the 74-station average are larger at large time lags than those at small time lags. It suggests that DFA can be robust in estimating the power-law scaling at STLB.

More precisely, for the lags in STLB (the corresponding high frequency band, HFB, in the CWT spectra), the average exponent is $4 / 3$ for the species $\mathrm{NO}, \mathrm{NO}_{2}, \mathrm{NO}_{\mathrm{x}}, \mathrm{CO}$, and $\mathrm{SO}_{2}$. The only exception is $\mathrm{O}_{3}$, which has an exponent of 2. For the lags in LTLB (the corresponding low frequency band, LFB, in the CWT spectra), the exponent is $3 / 4$, and is consistent for all the gases. Thus the dual-exponent structure is clearly established for DFA.

The DFA is able to identify the dual-exponent structure in the spectra with smaller standard deviations than those from the CWT (Fig. 3), but completely ignores the oscillatory aspect of the data. Although to show how the oscillation signals are "left" out in the operations is beyond the scope of this study, the running summations and the differencing in different lags, no doubt, somehow "smooth" the data to focus only on the scaling issues.

\subsection{Cross-evaluations of CWT and DFA spectra}

The purpose of this comparison between CWT and DFA exponents is to cross-evaluate the validity of the spectra obtained from two independent schemes. Here the exponents from CWT (Fig. 3) and DFA (Fig. 4) spectra of all stations are quantitatively estimated by the best linear fit over designated frequency bands. The scatter plots of the exponents 

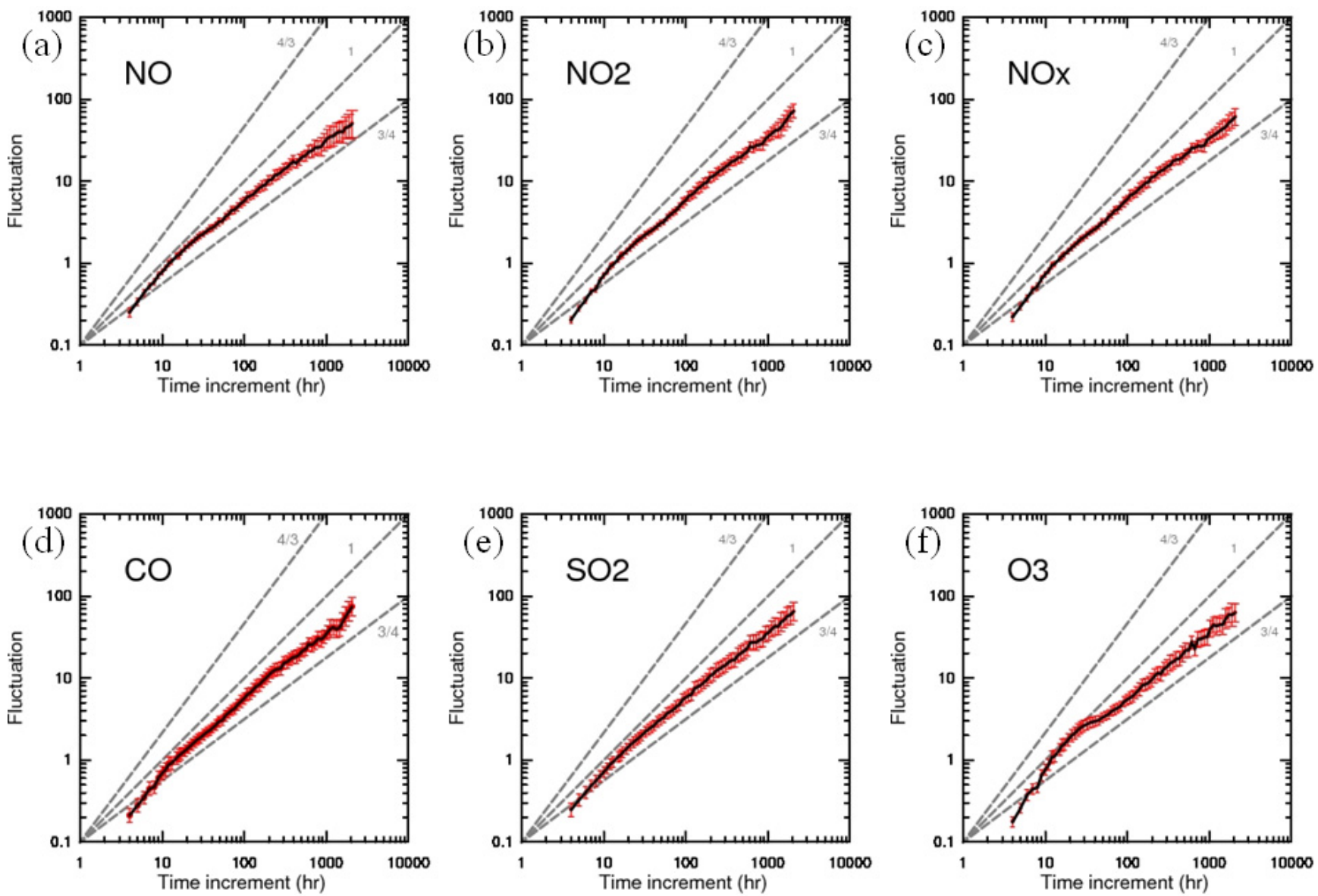

Fig. 4. The DFA spectra (in log-log scale) of the gases, $\mathrm{NO}$ (panel a), $\mathrm{NO}_{2}$ (panel b), $\mathrm{NO}_{\mathrm{x}}($ panel c), $\mathrm{CO}$ (panel d), $\mathrm{SO} 2$ (panel e), and $\mathrm{O}_{3}$ (panel f). The black curves are the 74-station averages, and the red bars are standard deviations from the averages. The grey dash lines indicate the power-law scaling of $4 / 3,1$ and $3 / 4$.

between the CWT spectra for both HFB and LFB and the DFA spectra for both STLB and LTLB are plotted in Fig. 5 and the exponent ranges are summarized in Table 1. For the species $\mathrm{NO}, \mathrm{NO}_{2}, \mathrm{NO}_{\mathrm{x}}, \mathrm{CO}$, and $\mathrm{SO}_{2}$, the scatter is quite consistent among themselves. For the LFB and LTLB (blue dots), the exponents are ranged between -0.5 and -1 for CWT and are between 0.75 and 1 for DFA. More interestingly, most of the exponents are projected in a line with a slope of -2 . For those of HFB and STLB (red dots), the exponents are in different ranges: The CWT spectra are between -1 and -2.5 , while the DFA's range is between 1 and 1.5. But they are still very close to the slope of -2 , although there is more scatter than those in LFB and LTLB. Such scatter is reflected in the sizes of the standard deviations in the CWT and DFA spectra (Figs. 3, 4).

Among the 6 gases, $\mathrm{O}_{3}$ is again different. So far, we know that its autocorrelation coefficients have the shortest time lag in the power-law scaling, its diurnal peak is the strongest, and its spectral slopes are the steepest for the high-frequency and the small time-lag bands in CWT and DFA, respectively.
Although the slopes are steep, their algebraic relationship between the CWT and DFA exponents remains (red dots, Fig. 5f). In other words, the relationship of the power-law exponents between CWT and DFA remains, even though the photochemical reactions involving $\mathrm{O}_{3}$ are volatile in $\mathrm{HFB}$ and STLB. For the LFB and LTLB (blue dots), the relationship of $\beta=2 \alpha-1$ is not well followed, and the standard deviations (the error bars) of the 74-station averages of the CWT and DFA exponents (Figs. 3f, 4f) are relatively larger than those of other gases.

The scatter of the CWT and DFA exponents may indicate other possibilities of the behavior of the chemical species in the lower atmosphere. One of them is the intensity of the species concentrations measured. The intensity can be affected by the emission, chemical reactions and meteorological conditions. The detailed analysis is beyond the scope of this paper, but an analysis of possible relationship between exponents described here and other statistical measures may shed further insight. 

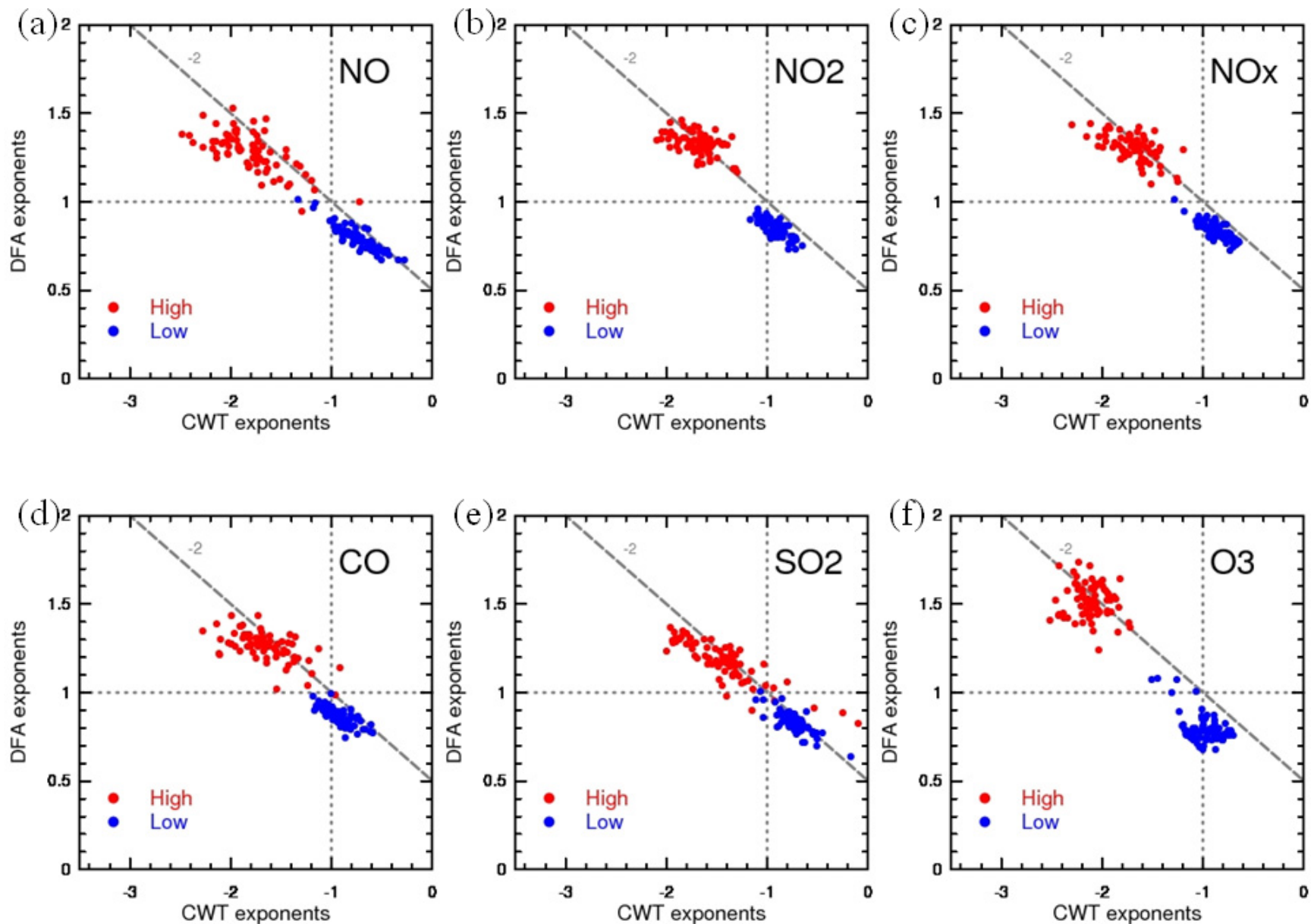

Fig. 5. The scatter plots of CWT and DFA exponents for the high-frequency band (red dots) and low-frequency band (blue dots) for the gases, $\mathrm{NO}$ (panel a), $\mathrm{NO}_{2}$ (panel b), $\mathrm{NO}_{\mathrm{x}}$ (panel c), $\mathrm{CO}$ (panel d), $\mathrm{SO}_{2}$ (panel e), and $\mathrm{O}_{3}$ (panel f). The grey dash lines have a slope of -2 .

Over the island of Taiwan, one of the interesting facts is that the intensity of different air pollutants varies from locations to locations. They are urban and rural, coastal and inland, plain and hill, and industrial and agricultural areas. Some basic statistical measures may be able to distinguish the differences in intensity among them. Both mean and standard deviation of the time series are selected to represent the strength in intensities. Then one would naturally expect, at least, some dependence of the spectra in terms of these measures.

Both CWT and DFA exponents may have some dependence on the potential spatial inhomogeneities, and they are compared with the averages and standard deviations from the time series at measurement stations that exponents are calculated. The corresponding averages and standard deviations are then normalized by the overall maxima of the averages and standard deviations, respectively. Thus, the ranges of averages and standard deviations of normalized intensity are between zero and one. Figures 6 and 7 depict the relationships between exponents and normalized averages and between exponents and normalized standard deviations for six gases, respectively. Exponents from high- and low-frequency bands (HFB and LFB) of CWT spectra and those from small and large time-lag bands (STLB and LTLB) of DFA are presented separately for each gas. All the exponents with a constant value spread over most of the ranges of normalized averages and standard deviations, except CO. Only the CWT HFB exponents of CO (Figs. 6d, 7d) show a weak correlation between the exponent and average/standard deviation: The larger the averages and standard deviations, the steeper the exponents. From this analysis, such spatial inhomogeneities do not affect the spectral exponents very much in terms of averages and standard deviations, except CO. Note that it is much harder to observe the correlations between DFA exponents and normalized averages/standard deviations. In other words, the universal structure of DFA exponents is more robust than that of CWT exponents.

Although the insensitivity of the exponents to the spatial inhomogeneities may be unexpected, the universal structures of CWT and DFA exponents provide an interesting opportunity to consider the possible parameterizations of chemical gas variability spectrally, at least for high 

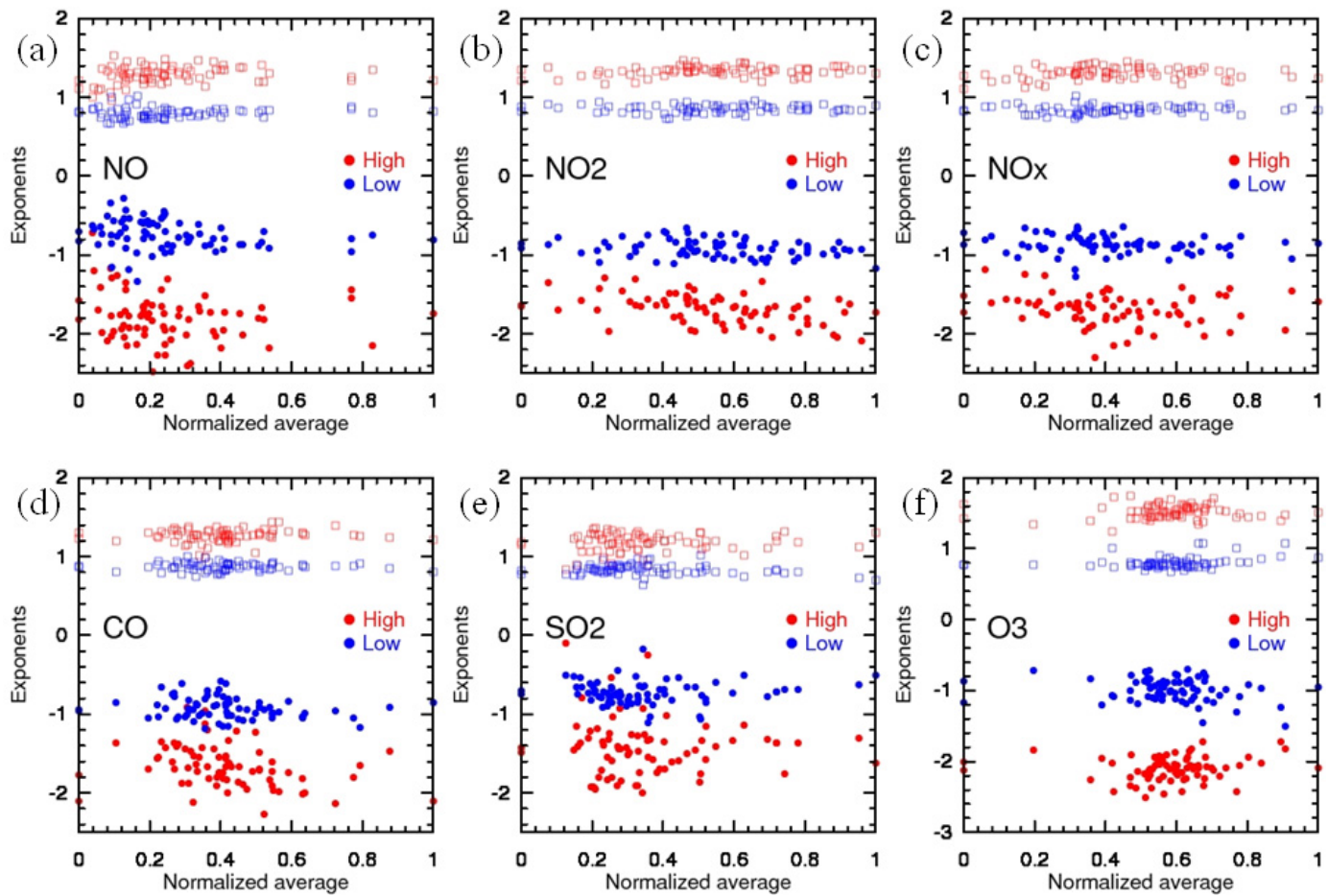

Fig. 6. The relationships between exponents and normalized averages for $\mathrm{NO}$ (panel a), $\mathrm{NO}_{2}$ (panel b), $\mathrm{NO}_{\mathrm{x}}\left(\right.$ panel c), $\mathrm{CO}$ (panel d), $\mathrm{SO}_{2}$ (panel e), and $\mathrm{O}_{3}$ (panel f), respectively. Exponents from high- and low-frequency bands (HFB (red dots) and LFB (blue dots)) of CWT spectra and those from small and large time-lag bands (STLB (red squares) and LTLB (blue squares)) of DFA are presented separately for each gas.
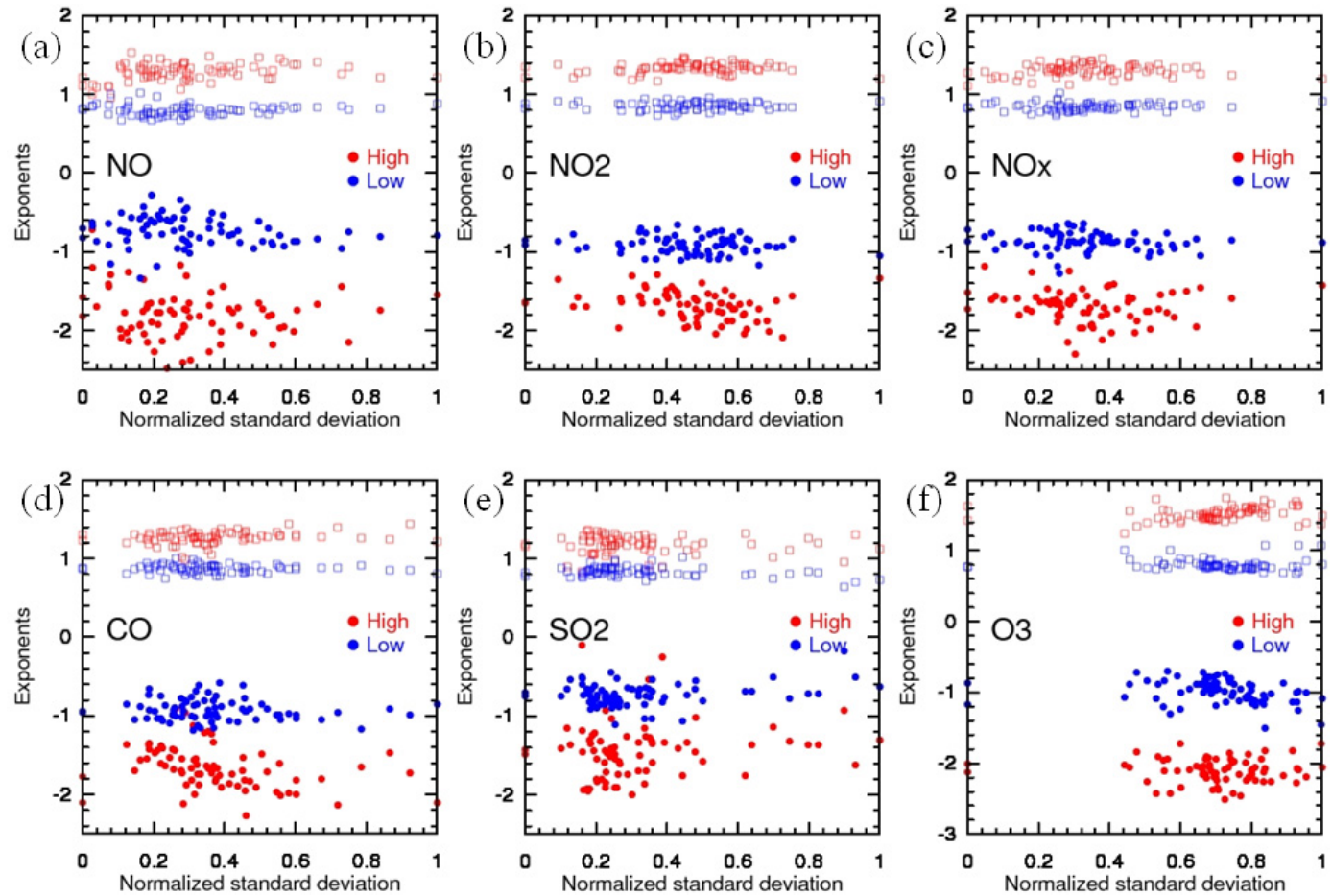

Fig. 7. The relationships between exponents and normalized standard deviations for $\mathrm{NO}$ (panel a), $\mathrm{NO}_{2}$ (panel b), $\mathrm{NO}_{\mathrm{x}}$ (panel c), $\mathrm{CO}$ (panel d), $\mathrm{SO}_{2}$ (panel e), and $\mathrm{O}_{3}$ (panel f). Exponents from high- and low-frequency bands (HFB (red dots) and LFB (blue dots)) of CWT spectra and those from small and large time-lag bands (STLB (red squares) and LTLB (blue squares)) of DFA are presented separately for each gas. 

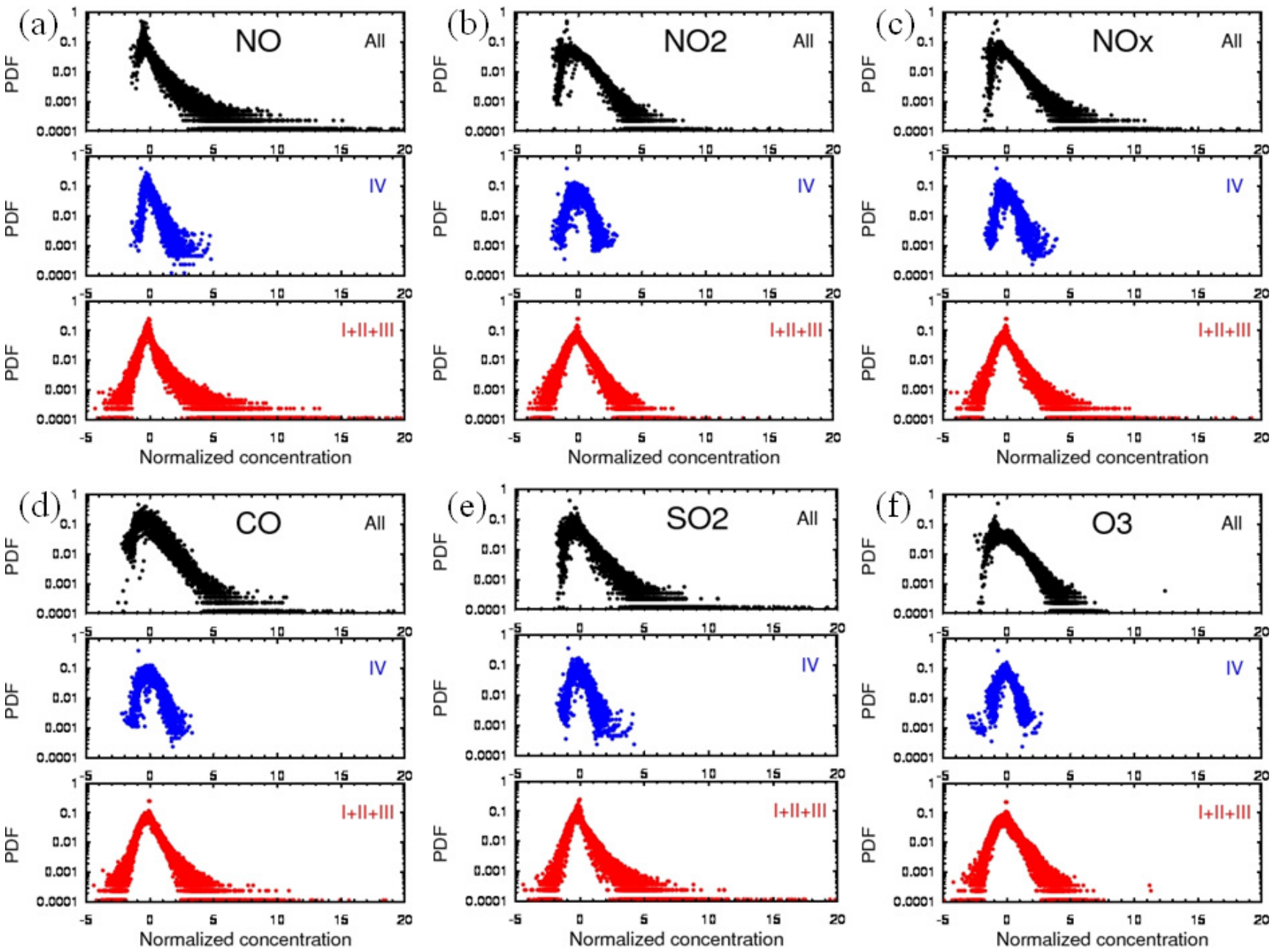

Fig. 8. The PDFs for the gases, $\mathrm{NO}$ (panel a), $\mathrm{NO}_{2}$ (panel b), $\mathrm{NO}_{\mathrm{X}}$ (panel c), $\mathrm{CO}$ (panel d), $\mathrm{SO}_{2}$ (panel e), and $\mathrm{O}_{3}$ (panel f) plotted in the semi-log scale. For each gas, the black, blue, and red dots are for the original time series, the reconstructed time series in the frequency bands IV and V, respectively. The bands IV and V include the frequencies less than and larger than the synoptic peak, respectively.

frequencies and small time-lags. Furthermore, no direct evidence claims that the dominant $-5 / 3$ exponent in the CWT high-frequency band is the result of local turbulence, but strong implication is suggested by the analysis presented.

\subsection{PDFs: original and reconstructed}

All the gases considered here are naturally positive definite, and have their own special spectral characteristics. The classical Gaussian distribution usually cannot represent their variability. In the following, their statistical regularity is investigated in terms of the normalized frequency distribution, i.e., the probability distribution function (PDF). Furthermore, based on their spectral structures resulted from CWT, the time series are reconstructed at each characteristic spectral band. Then their PDFs illustrate their statistical regularities.
First, the original PDFs of all the gases are depicted in the top panels of Figs. 8a-f for each gas. They all positively skewed, and their skewness presented in Fig. 11a show a range from at least 1 to greater than 4 . Their kurtoses are also large (Fig. 11b). Both $\mathrm{NO}$ and $\mathrm{SO}_{2}$ have relatively large skewness and kurtosis, in comparison with the others. Also noted is that all of them have heavy tails, only on the positive side.

Then after the time series are decomposed by CWT, the spectral coefficients are grouped for the spectral bands of frequencies greater than the semi-diurnal (I), the semi-diurnal and diurnal frequencies (II), the frequencies less than the diurnal but greater than the synoptic (III), and the frequencies less than the synoptic (IV). The bands I and III are the same as the HFB and LFB, respectively, and their spectral variances display almost constant power-law exponents (Fig. 3). The band V has all the spectral coefficients in the bands I, II and III. In other words, the bands IV and V represent the 

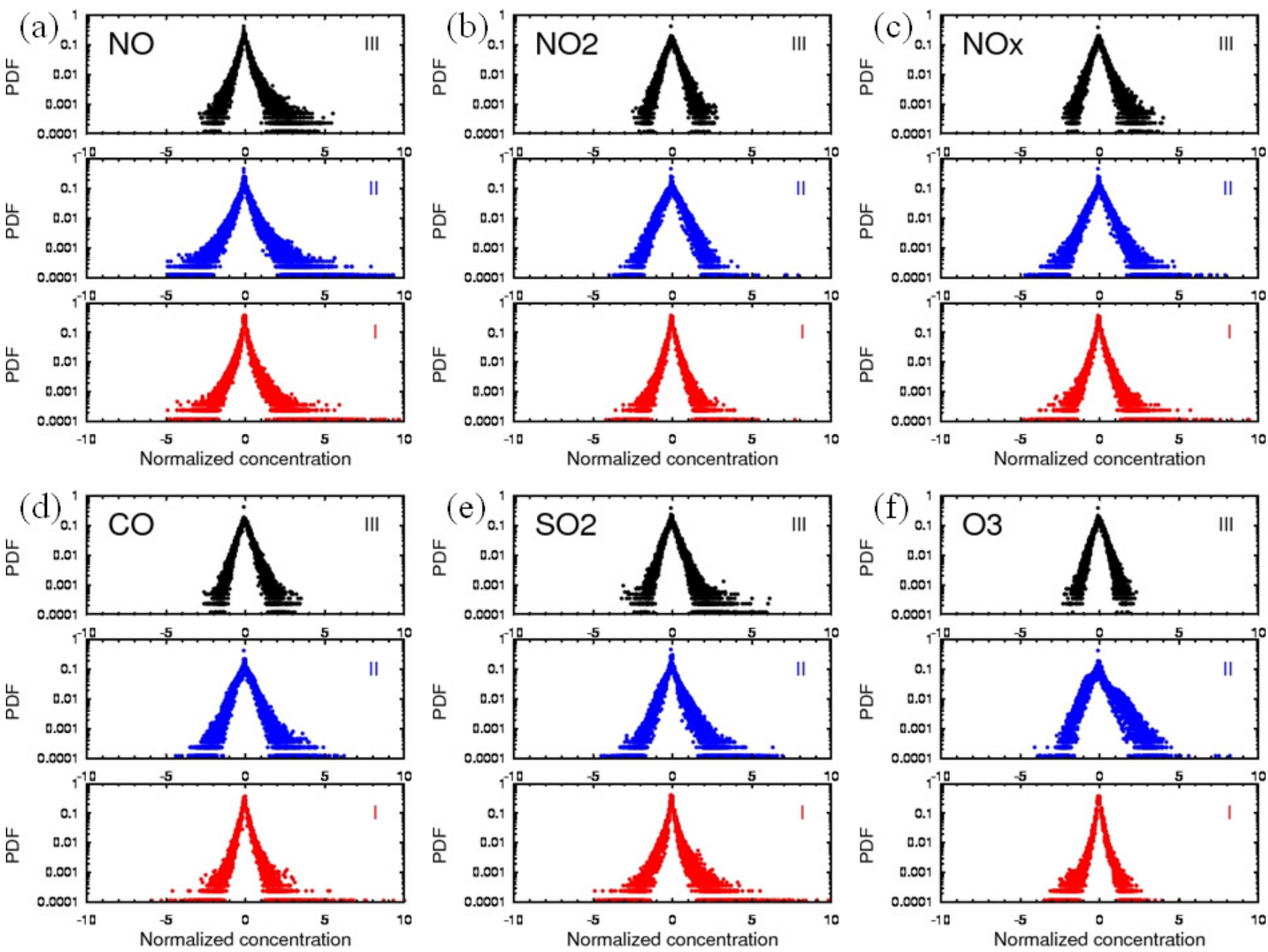

Fig. 9. The PDFs for the gases, $\mathrm{NO}$ (panel a), $\mathrm{NO}_{2}$ (panel b), $\mathrm{NO}_{\mathrm{x}}$ (panel c), $\mathrm{CO}$ (panel d), $\mathrm{SO}_{2}$ (panel e), and $\mathrm{O}_{3}$ (panel f) plotted in the semi-log scale. For each gas, the black, blue, and red dots are for reconstructed time series in the frequency bands III, II and I, respectively. The sum of the frequencies in regions I, II and III is the same as those in the region V shown in Fig. 8.

signals in low and high frequencies, respectively. Figure 8a$\mathrm{f}$ display PDFs for six gases. For each gas, the original PDF (top panel), the low-frequency PDF (band IV; middle panel), and the high-frequency PDF (band V; bottom panel) are shown in black, blue, and red, respectively.

The low-frequency PDFs (band IV) generally have not only rather weak skewness but also close to zero kurtosis (see also Fig. 11). The presence of the heavy tails is extremely minimal. On the other hand, the high-frequency PDFs (band V) have quite similar skewness and kurtosis patterns of the original PDFs for all gases. Moreover, the PDFs are getting more symmetric than the original PDFs, and the positive heavy tails get much more significant than those of the original PDFs. It suggests that signals in the high frequencies are definitely non-Gaussian (large positive skewness and large kurtosis), but those in the low frequencies are really not so, still not Gaussian.
All the signals in the band $\mathrm{V}$ are further separated into three bands, I, II and III, and their PDFs are presented in Fig. 9. They all give a symmetric sense, i.e., relatively low skewness, particularly the PDFs of the bands II and III (see also Fig. 11). For kurtosis, the values are much larger for the signals of the band I than those of the bands II and III. Furthermore, the PDFs with small kurtosis are $\Lambda$-shaped and less cusp, but for those with large kurtosis, the larger the kurtosis and the more cusp-shaped the PDFs. The cusp-shaped PDFs in the semi-log plots as shown in Fig. 9 have actually the power-law shape in the log-log plots. Figure 10 displays the log-log plots of the positive side of the PDFs shown in Fig. 9. On the heavy-tail side, a power-law scaling of -3 is suggested (the dashed line) for most of the PDFs of the bands I and III. But such scaling is less evident for those of the band II, probably because the CWT spectra have power-law scaling only in bands I and III, but not in the band II. In fact, the $\Lambda$-shaped PDFs indicate the exponential distributions. 

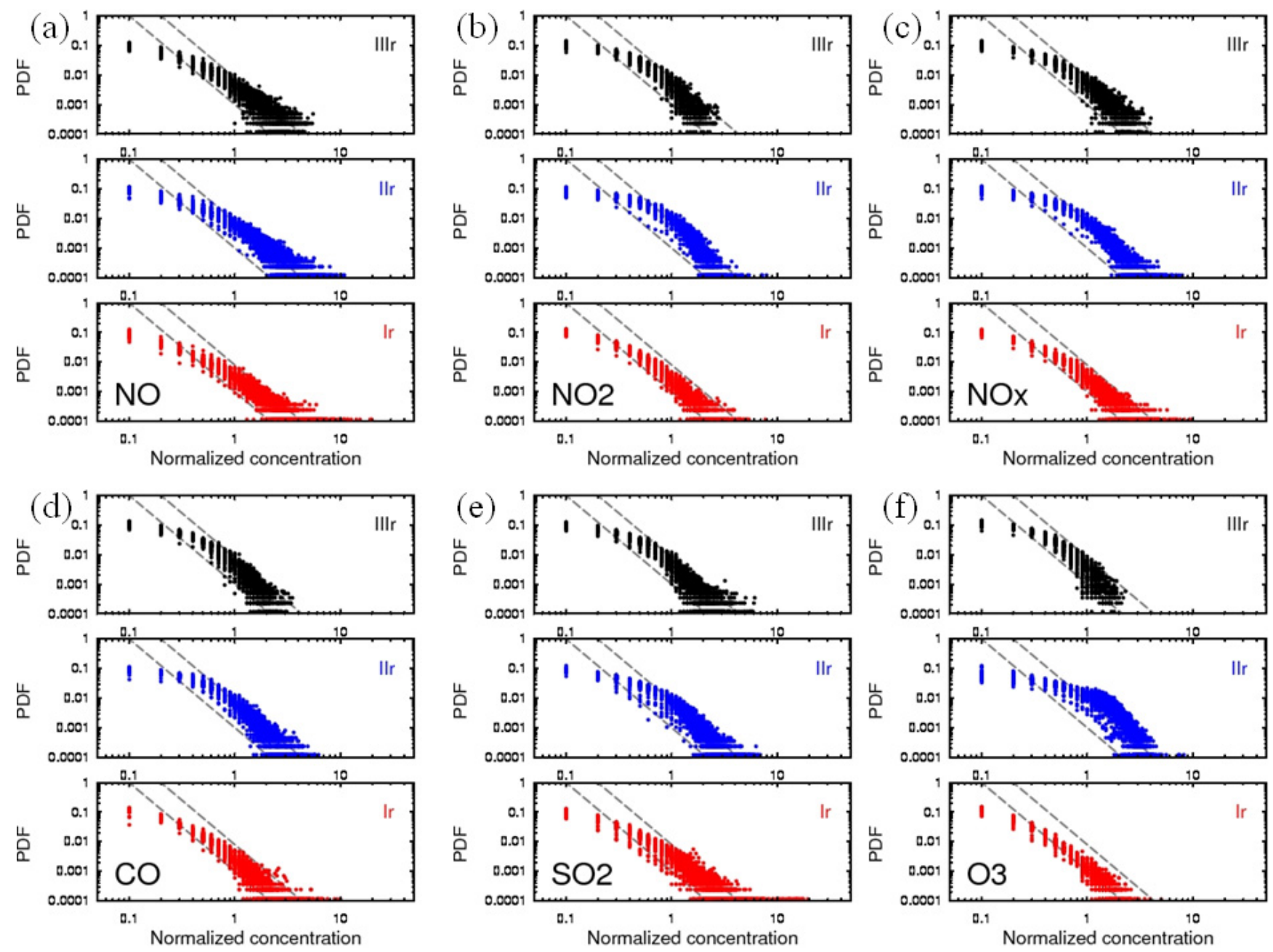

Fig. 10. Same as in Fig. 9 except only the positive sides of the PDFs plotted in the log-log scale. The grey dash line has an exponent of -3 .

Based on the spectra from CWT and DFA, the time series are first decomposed and reconstructed selectively to produce the time series for certain frequency bands. The most interesting among them is the cusp-shaped PDFs of the time series from the high-frequency band in the semi-log plot (Fig. 9). And they are definitely non-Gaussian. As a matter of fact, the cusp-shape in the semi-log plot can be approximated as the power-law scaling in the log-log plot (Fig. 10). For the relatively large intensity, the scaling is about -3 , and indicates the heavy tail. This is another important factor, other than the universal exponents, to consider/constrain possible parameterizations for the high frequencies and/or small scales.

\section{Aerosol spectra and PDF}

Atmospheric aerosols are ubiquitous in the air and often observable as dust, smoke and haze, and predominantly derive from natural sources, sea salt, dust, and biogenic particles. Primary aerosols are emitted directly while secondary aerosol matter forms in the atmosphere from gas to parti- cle conversion. Anthropogenic sources, such as from a variety of combustion processes, are also abundant. The presence of aerosol in the atmosphere causes various "direct" and "indirect" effects in our climate system. Aerosols affect Earth' energy budget by scattering and absorbing radiation (the "direct effect") and by modifying amounts and microphysical and radiative properties of clouds (the "indirect effect"). Aerosols influence cloud properties through their role as cloud condensation nuclei and/or ice nuclei. Not only do chemical reactions but also physical processes influence the compositions, and structures of number density and mass concentration of aerosols. In order to understand atmospheric aerosols, the knowledge of particle properties such as their size, shapes, chemical compositions, their surfaces, their optical properties, and their volumes and masses has advanced significantly in recently decades. At 74 air quality monitoring stations over the island of Taiwan (Fig. 1), the mass concentrations of particulate matter were measured for all bin sizes less than $10 \mu\left(\mathrm{PM}_{10}\right)$. 

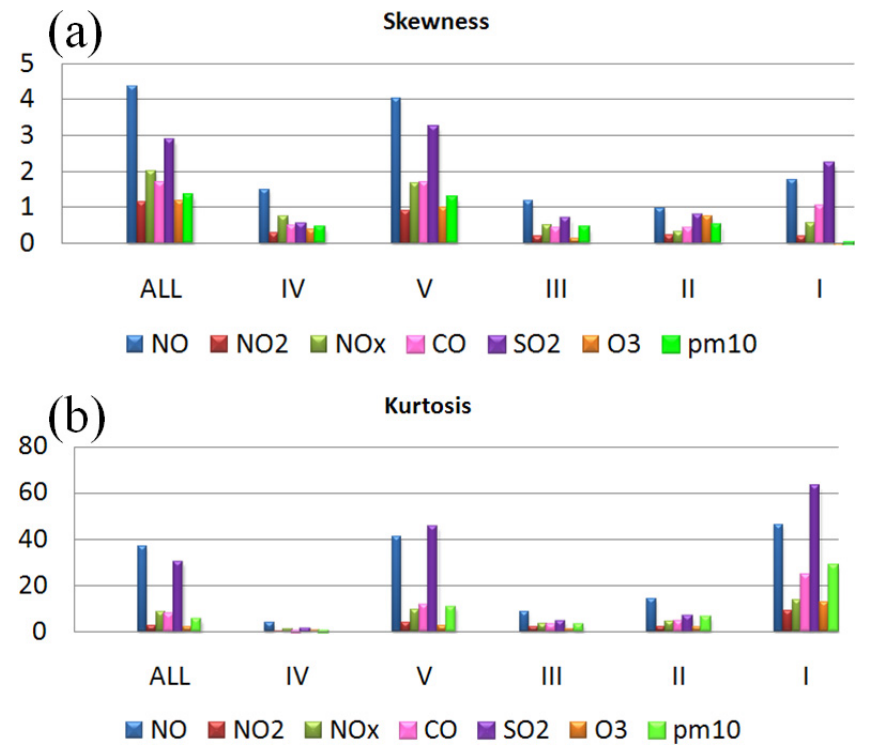

Fig. 11. For of the gases $\left(\mathrm{NO}, \mathrm{NO}_{2}, \mathrm{NO}_{\mathrm{x}}, \mathrm{CO}, \mathrm{SO}_{2}\right.$, and $\left.\mathrm{O}_{3}\right)$ and aerosol $\left(\mathrm{PM}_{10}\right)$, the skewness (panel $\mathbf{a}$ ) and kurtosis (panel $\mathbf{b}$ ) of the original time series (ALL) and the reconstructed time series for the frequency bands of IV, V, III, II, and I.

The autocorrelation coefficients of the $\mathrm{PM}_{10}$ time series also show a power-law scaling of about $-1 / 3$ for the first 24 hour lag, before the diurnal oscillation takes over (Fig. 12a). Its pattern is quite similar to those of the gases examined in Fig. 2 except $\mathrm{O}_{3}$.

Again, the CWT spectra of the $\mathrm{PM}_{10}$ (Fig. 12b) have a pattern similar to the gas spectra described in the last section. Other than the diurnal and semi-diurnal, and intermediate peaks, the synoptic peak is very weak and the weekly is missing. But, two power-law scaling regions in HFB and LFB have the average exponents of -2.5 and -1 , respectively. Appearing at the high-frequency end of the spectra, the 74-station averaged spectra have relatively large standard deviations, which increase as the frequency increases toward the limit of the resolution, 2 hours. The DFA spectra of the $\mathrm{PM}_{10}$ time series are displayed in Fig. 12d. The mean exponent follows 4/3 in STLB up to the diurnal time-lag, and then transits to gentler exponent toward 3/4 at the very large time-lag.

For comparing the CWT and DFA exponents, the scatter plot (Fig. 12c) shows that the slope of the algebraic relationship of $\beta=2 \alpha-1$ is roughly followed. For the CWT's HFB and the DFA's STLB, the exponents range between -1.5 and -3 , and between 1 and 1.7, respectively. The spread in this range is the largest, compared with those gases in Fig. 5, but the spread is quite small, focusing at -1 and 1 for the CWT's LFB and DFA's LTLB, respectively. Such a large spread in the CWT's HFB is consistent with the large error bars showing in the average CWT spectrum.
For the issue of the potential spatial inhomogeneities for the $\mathrm{PM}_{10}$ time series, each $\mathrm{PM}_{10}$ 's average and standard deviation of each measurement station are first computed, and then the overall maxima of them are used to normalize each average and standard deviation, respectively. The relationship between exponents and their respective normalized averages and standard deviations are shown in Fig. 13. For the CWT's LFB and DFA's LTLB, no clear trend is noted. However, a trend may be seen for the CWT's HFB and DFA's STLB, but weak. This result is similar to that of CO (Figs. 6d, 7d). For the purpose of considering/constraining possible parameterizations, the linear relationships between the exponents and the averages/standard deviations can still be useful, if they can be established, but this waits for further analysis. The potential spatial inhomogeneities issue for $\mathrm{CO}$ may be considered similarly.

The PDF of $\mathrm{PM}_{10}$ and its PDFs over different frequency bands are displayed in Fig. 14. Figure 14a contains the original PDF, and PDFs over low (band IV) and high (band V) frequencies. Visually, the $\mathrm{PM}_{10}$ PDF patterns are similar to those of its gaseous counterparts (Fig. 8). In terms of skewness and kurtosis (Fig. 11), $\mathrm{PM}_{10}$ has high correlations with $\mathrm{O}_{3}$ and $\mathrm{NO}_{\mathrm{x}}$. The low-frequency PDF essentially has no tail, while the high-frequency one has a longer heavy tail on the positive side.

The high-frequency PDF over the band $\mathrm{V}$ is further separated into bands of I, II, and III (Fig. 14b). The PDF of the highest frequency band (I) is not only symmetric (low skewness) but also very cusp (high kurtosis), and those from the bands II and III depict the $\Lambda$-shapes. The comparisons of these skewness and kurtosis with those of chemical gases are shown in Fig. 11. These high-frequency PDFs are further plotted in the log-log scale (Fig. 14c) to show the power-law scaling of -3 at large normalized concentrations for the band I, while other PDFs in the bands II and III have steeper slopes towards exponential. (In this panel, only the right sides of the PDFs are re-plotted from Fig. 14b.)

In summary, although aerosol $\left(\mathrm{PM}_{10}\right)$ has different physical characteristics than gas phase chemical species (NO, $\mathrm{NO}_{2}, \mathrm{NO}_{\mathrm{x}}, \mathrm{CO}, \mathrm{SO}_{2}$, and $\mathrm{O}_{3}$ ), its basic spectral structures and statistical regularity are similar to those of the gases.

\section{Summary and concluding remarks}

With the intent to gain further knowledge of surface atmospheric chemistry in terms of dynamic scales, a spectral and statistical analysis methodology has been introduced here. To demonstrate the capability of the methodology, the chemical gases $\left(\mathrm{NO}, \mathrm{NO}_{2}, \mathrm{NO}_{\mathrm{x}}, \mathrm{CO}, \mathrm{SO}_{2}\right.$, and $\left.\mathrm{O}_{3}\right)$ and aerosol $\left(\mathrm{PM}_{10}\right)$ measured at 74 air quality monitoring stations over the island of Taiwan are analyzed for the year of 2004 at the hourly resolution. They represent near-surface air quality of a variety of geographic settings 

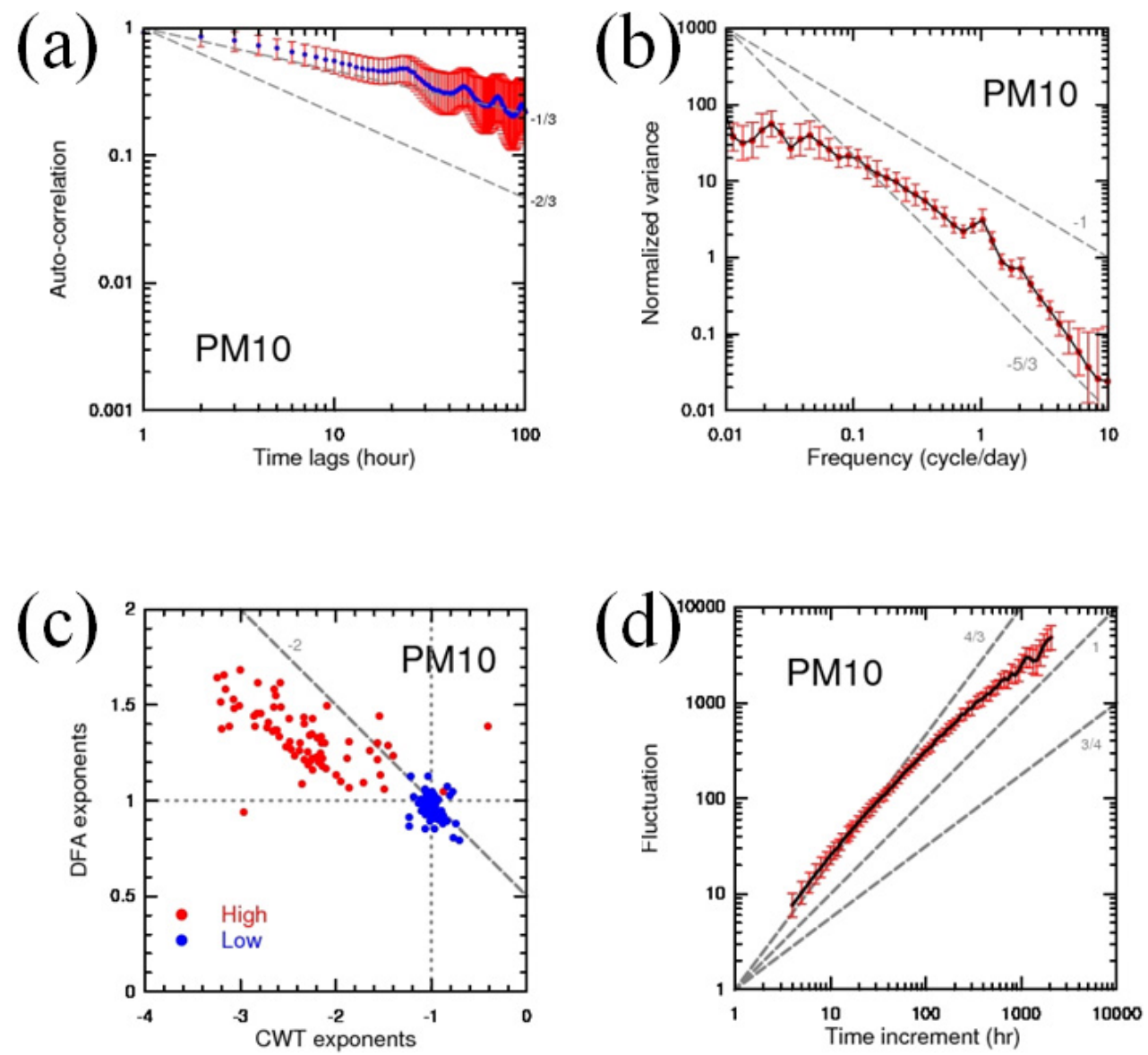

Fig. 12. For aerosol $\left(\mathrm{PM}_{10}\right)$. Panel (a): auto-correlation. Panel (b): CWT spectrum. Panel (d): DFA spectrum. Panel (c): the scatter plot of the CWT and DFA spectral exponents.
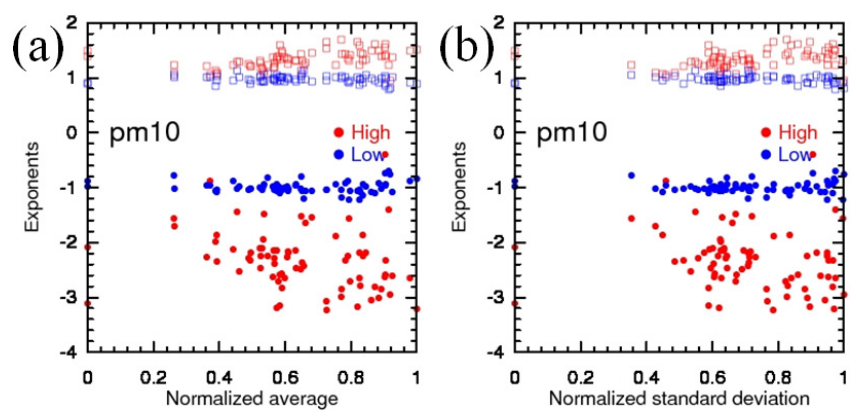

Fig. 13. The relationships between exponents and normalized averages (panel a) and between exponents and normalized standard deviations (panel b) for $\mathrm{PM}_{10}$, respectively. Exponents from highand low-frequency bands (HFB (red dots) and LFB (blue dots)) of CWT spectra and those from small and large time-lag bands (STLB (red squares) and LTLB (blue squares)) of DFA are presented.

and include urban/rural, coastal/inland, plain/hill, and industrial/agricultural locations. All the measured time series undergo spectral decompositions/reconstructions to reveal some of their common spectral and statistical characteristics. The major findings are as follows:

- Although the auto-correlations of all the gases and aerosol indicate power-law decay of exponents between $-1 / 3$ and $-2 / 3$, the time-lags are, in general, short (less than 24 hours), and ozone's lag is even shorter, only $3 \sim 4$ hours, because of its highly reactive nature. They all suggest significant diurnal oscillation though.

- All the chemical species, gases and aerosol, have distinct characteristics of spectral peaks from the continuous wavelet transforms (CWT). Various temporal peaks are identified in the average spectra from the 74 stations. The semi-diurnal peak is primarily caused by the morning and afternoon traffic emissions, and the photochemical and boundary-layer processes modulate the 

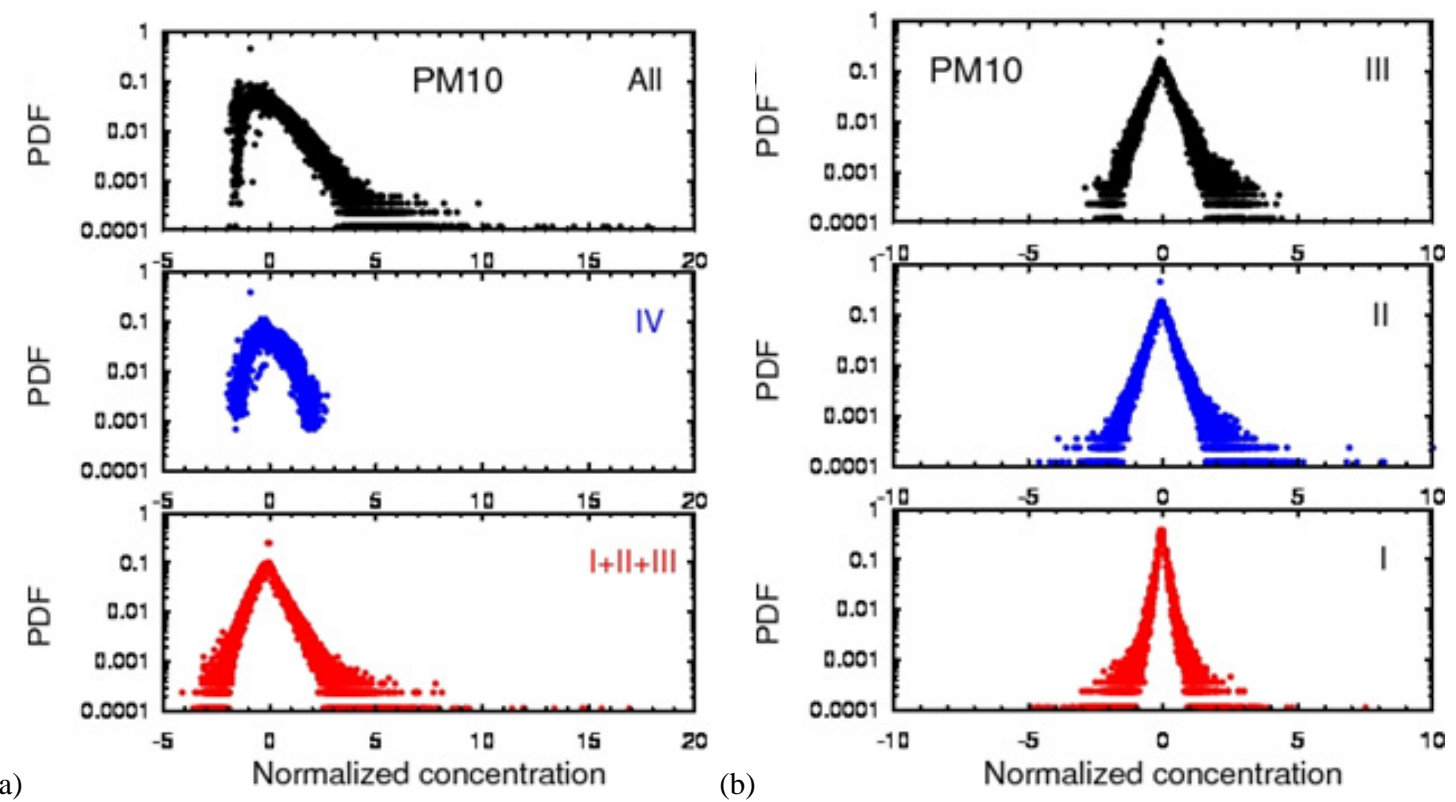

(a)
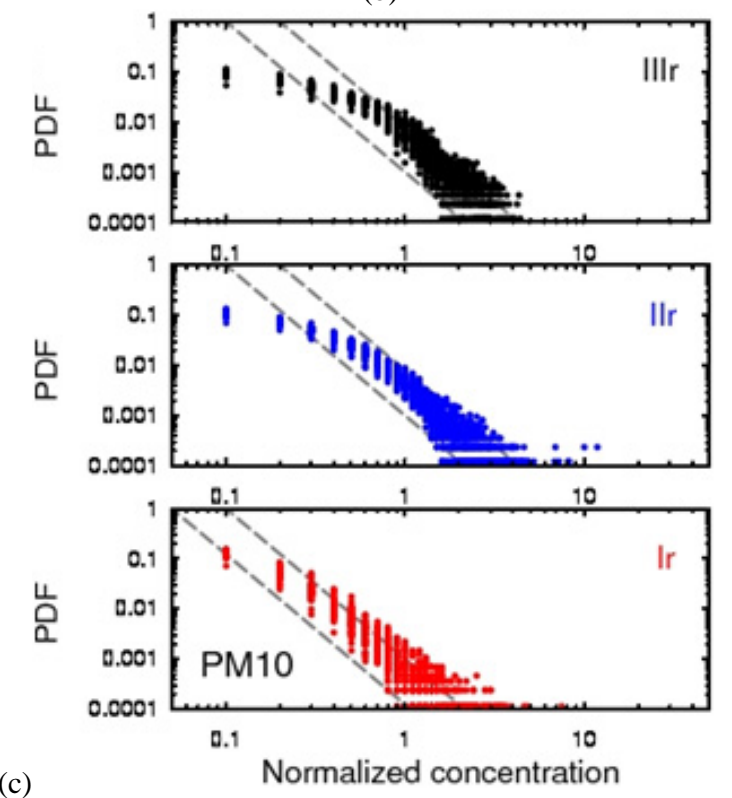

Fig. 14. PDFs for aerosol $\left(\mathrm{PM}_{10}\right)$. Panel (a): the black, blue, and red dots are for the original time series, the reconstructed time series in the frequency bands IV and V, respectively. The bands IV and V include the frequencies lower than and higher than the synoptic peak, respectively. Panel (b): the black, blue, and red dots are for reconstructed time series in the frequency bands III, II and I, respectively. The sum of the frequencies in regions I, II and III is the same as those in the region V. Panel (c): as in panel (b) except only the positive sides of the PDFs plotted in the log-log scale. The grey dash line has an exponent of -3 . In the panels (a) and (b), the plots are in semi-log scale.

diurnal peak. The weekly peak has been suggested as the low emissions of chemical species during weekends, but synoptic-scale disturbances have a similar temporal scale. The longer-period peaks, the intermediate of $20 \sim 30$ days and the intraseasonal of $30 \sim 100$ days could be related to longer-period transient weather features, such as tropical cyclones, cold-air outbreaks, and organized tropical convection. As the island of Taiwan is situated in the subtropics, the interactions be- tween extratropical and tropical weather at different scales from season to season are plausible for those broad-band signals. Although the upper bound of the intraseasonal band of $30 \sim 100$ day may need multiyear data to confirm its statistical significance, physically this broad peak relates to the tropical MaddenJulian Oscillation quite well. Then long-range transport of those chemical species also can play a critical role. The connection between the local air quality and 
varying transient weather is important for understanding the air chemistry in the lower atmosphere, and further investigations are warranted to clarify these broader peaks.

- The CWT dual-exponent spectral characteristics for all the gases and aerosol are consistent with those obtained independently from the detrended fluctuation analysis (DFA), and they follow the algebraic relationship, $\beta=2 \alpha-1$, where $\beta$ and $\alpha$ are the exponents of CWT and DFA spectra, respectively. Generally, two different regimes can be identified by different exponents separated by the diurnal/semi-diurnal peaks. The average slope of $-5 / 3$ for the high-frequency band (HFB) in the CWT spectra (varying from -1 to $-5 / 2$ ) corresponds to that of $4 / 3$ for the short time-lag band (STLB) in the DFA spectra (varying from 1 to $3 / 2$ ). On the other hand, for the low-frequency band (LFB) in the CWT spectra and the corresponding long time-lag band (LTLB) in the DFA spectra, the exponents vary from $3 / 4$ to 1 and from $-1 / 2$ to -1 , respectively. Thus, the measured data is a combination of stationary and non-stationary. In other words, the signals are stationary for the LFB and LTLB, but are non-stationary for the HFB and STLB, in average.

- In general, the dual-exponent characterizations are realized in both CWT and DFA spectra as summarized in Table 1 . Of course, many possible processes are responsible for the variability of the exponents, and should be identified to further understand such variability. Further identifications can also help to formulate/constrain accurate parameterizations for the unresolved processes.

- Traditionally, a sequence of data is characterized by the statistical measures of the mean, standard deviation, skewness and kurtosis for the data's normalized frequency distribution, i.e., the probability distribution function (PDF). Because of the positive definiteness of the chemical data, the PDFs are expected to be positively skewed, even if the data are normalized. Based on the spectra from CWT and DFA, the time series are first decomposed and reconstructed selectively to produce the time series for certain frequency bands. The most interesting among them is the cusp-shaped PDFs of the time series from the high-frequency band in the semi-log plot. And they are definitely non-Gaussian. As a matter of fact, the cusp-shape in the semi-log plot can be approximated as the power-law scaling in the log$\log$ plot. For the relatively large intensity, the scaling is about -3 , and indicates the heavy tail.

Our average CWT spectral exponent of $-5 / 3$ in the highfrequency band obtained from the chemical data has the same exponent of the Kolmogorov's turbulent spectrum, and is hypothesized that the high-frequency activity in the chemical data is strongly related to the local turbulence. There is a major difference between Kolmogorov's and our spectra, and that is the former is valid at the temporal scale of seconds, and our data resolution is only one hour. Gage (2004) gave a thorough review on the mesoscale variability of $-5 / 3$ scaling. Our scaling law is relevant to the mesoscale variability. Local smaller-scale turbulence could be universal, but largerscale dynamic features vary from time to time and from place to place. Further studies are required to explore such linkages between chemistry and turbulence in small scales.

One of the objectives of this investigation is to identify some spectral structures and statistical regularities among different chemical species, gas and aerosol, at different geographic locations in different seasons. Should such spectral structures and statistical regularities have some commonalities, they can be formulated for and/or constrain parameterizations, particularly for the unresolved chemical processes in numerical simulations and forecasts of chemical weather. Kolmogorov's power-law scaling of turbulence established the modern view of small-scale activity in the inertial subrange, and provided a new approach to model the turbulent process in the range where the sub-grid-scale has not been able to be resolved. The modern large-eddy simulation (LES) methodology is based on such foundation.

Another important and interesting revelation from the data is the consistent characterization of the power-law scaling in the PDFs based on the frequency-based reconstructions. For the high-frequency band, the PDFs from the reconstructed time series consistently have cusp-shape in the semi-log plots (Figs. 9, 14), suggesting high kurtosis (Fig. 11b). Although the original data have relatively high kurtosis, such high kurtosis with low skewness only systematically appears in the reconstructed data from the high-frequency band. In fact, they all have the power-law scaling of same exponent of -3 over their heavy tails. This statistical regularity is especially useful, because the unresolved chemical/physical processes at high frequencies can be parameterized, and/or constrained, based on the statistical analysis of the observed data over a long period of time. In this investigation, the data have an hourly resolution for a year.

Here, we have revealed not only the consistent characteristics in both CWT and DFA spectra, but also the distinct power-law scaling in the PDFs of the reconstructed signals from the CWT spectra in the high-frequency band. The same analysis methodology for the spectral and PDF structures described in this paper can also be applied to the results from numerical experiments such that objective evaluations of experiments can be performed. The advantage of this methodology is that the results can be assessed in terms of dynamic scales, in comparison with the structures from observations/measurements. So far the analyses are applied only in time, but it is straightforward to extend the same methodology to space in higher dimensions.

The variability of the exponents in CWT and DFA spectra, shown as the sizes of the standard deviations (Figs. 3, 4, 
12) and the exponent scatters (Figs. 5, 12) can be the consequences of various chemical/physical processes under various conditions, such as urban/rural, coastal/inland, plain/hill and industrial/agricultural locations. However, in this analysis most of the chemical exponents exhibited are independent of the concentration averages and standard deviations, based on the data from 2004 alone. The seasonality could be another issue. Multi-year data are necessary for this purpose. The exponents can be classified accordingly for further studies. Such investigations will further our knowledge of the detailed processes underlying the parameterizations, and provide more accurate classifications of parameterizations.

Acknowledgements. Discussions with Rob Frehlich, Adrian Tuck, and Christine Wiedinmyer have been very much appreciated. We gratefully acknowledge the review comments from Zhong-Wei Yan and an anonymous reviewer. $\mathrm{HH}$ has enjoyed the hospitality and support provided by the Research Center for Environmental Changes, Academia Sinica when he visited AC/RCEC. The National Center for Atmospheric Research is operated by the University Corporation for Atmospheric Research under sponsorship from the National Science Foundation. CYL acknowledges the support from the National Research Council (NSC 99-2111-M001-007-MY3).

Edited by: L. Molina

\section{References}

Bachmeier, A. S., Newell, R. E., Shipham, M. C., Zhu, Y., Blake, D. R., and Browell, E. V.: PEM-West A: Meteorological overview, J. Geophys. Res., 101, 1655-1677, 1996.

Buldyrev, S., Goldberger, A. L., Havlin, S., Mantegna, R. N., Masta, M. E., Peng, C.-K., Simons, M., and Stanley, H. E.: Long-range correlation properties of coding and noncoding DNA sequences: GenBank analysis, Phys. Rev. E., 51, 5084-5091, 1995.

Cho, J. Y. N., Zhu, Y., Newell, R. E., Anderson, B. E., Barrick, J. D., Gregory, G. L., Sachse, G. W., Carroll, M. A., and Albercook, G. M.: Horizontal wavenumber spectra of winds, temperature, and trace gases during the Pacific Exploratory Missions, 1, Climatology, J. Geophys. Res., 104, 5697-5716, 1999a.

Cho, J. Y. N., Newell, R. E., and Barrick, J. D.: Horizontal wavenumber spectra of winds, temperature, and trace gases during the Pacific Exploratory Missions: 2. Gravity waves, quasitwo-dimensional turbulence, and vortical modes, J. Geophys. Res., 104, 16297-16308, 1999b.

Emmons, L. K., Walters, S., Hess, P. G., Lamarque, J.-F., Pfister, G. G., Fillmore, D., Granier, C., Guenther, A., Kinnison, D., Laepple, T., Orlando, J., Tie, X., Tyndall, G., Wiedinmyer, C., Baughcum, S. L., and Kloster, S.: Description and evaluation of the Model for Ozone and Related chemical Tracers, version 4 (MOZART-4), Geosci. Model Dev., 3, 43-67, doi:10.5194/gmd3-43-2010, 2010.

Farge, M.: Wavelet transforms and their applications to turbulence, Annu. Rev. Fluid Mech., 24, 395-457, 1992.

Fast, J. D., Gustafson Jr., W. I., Easter, R. C., Zaveri, R. A., Barnard, J. C., Chapman, E. G., Grell, G. A., and Peckham, S. E.: Evolution of ozone, particulates, and aerosol direct ra- diative forcing in the vicinity of Houston using a fully-coupled meteorology-chemistry-aerosol model, J. Geophys. Res., 111, D21305, doi:10.1029/2005JD006721, 2005.

Gage, K. S.: Dynamic processes contributing to the mesoscale spectrum of atmospheric motions, Atmospheric Turbulence and Mesoscale Meteorology, edited by: Fedorovich, E., Rotunno, R., and Stevens, B., Chap. 10, 223-263, 2004.

Gage, K. S. and Nastrom, G. D.: Theoretical interpretation of atmospheric wavenumber spectra of wind and temperature observed by aircraft during GASP, J. Atmos. Sci., 43, 729-740, 1986.

Grell, G. A., Peckham, S. E., Schmitz, R., McKeen, S. A., Frost, G., Skamarock, W. C., and Eder, B.: Fully coupled online chemistry within the WRF model, Atmos. Environ., 39, 6957-6975, 2005.

Güsten, H., Heinrich, G., and Sprung, D.: Nocturnal depletion of ozone in the Upper Rhine Valley, Atmos. Environ., 32, 11951202, 1998.

Hastie, D. R., Shepson, P. B., Sharma, S., and Schiff, H. I.: The influence of the nocturnal boundary layer on secondary trace species in the atmosphere at Dorset, Ontario, Atmos. Environ., 27, 533-541, 1993.

Hausdorff, J. M., Purdon, P. L., Peng, C. K., Ladin, Z., Wei, J. Y., and Goldberger, A. L.: Fractal dynamics of human gait: Stability of long-range correlations in stride interval fluctuations, J. Appl. Physiol., 80, 1448-1457, 1996.

Heneghan, C. and McDarby, G.: Establishing the relation between detrended fluctuation analysis and power spectral density analysis for stochastic processes, Phys. Rev. E, 62, 6103-6110, 2000.

Hsu, H., Moncrieff, M. W., Tung, W.-w., and Liu, C.: Multiscale temporal variability of warm-season precipitation over North America: Statistical analysis of radar measurements, J. Atmos. Sci., 63, 2355-2368, 2006.

IPCC: Climate Change 2001: The Science Basis, Contribution of Working Group I to the Third Assessment Report of the Intergovernmental Panel on Climate Change, Cambridge University Press, Cambridge, United Kingdom and New York, NY, USA, 881 pp., 2001

IPCC: Climate Change 2007: The Physical Science Basis. Contribution of Working Group I to the Fourth Assessment Report of the Intergovernmental Panel on Climate Change, edited by: Solomon, S., Qin, D., Manning, M., Chen, Z., Marquis, M., Averyt, K. B., Tignor, M., and Miller, H. L., Cambridge University Press, Cambridge, United Kingdom and New York, NY, USA, 996 pp., 2007.

Jolliffe, I. T. and Stephenson, D. B.: Forecast Verification: A Practitioner's Guide in Atmospheric Science, John Wiley, 240 pp. 2003.

Kalnay, E., Kanamitsu, M., Kistler, R., Collins, W., Deaven, D., Gandin, L., Iredell, M., Saha, S., White, G., Woollen, J., Zhu, Y., Leetmaa, A., Reynolds, R., Chelliah, M., Ebisuzaki, W., Higgins, W., Janowiak, J., Mo, K. C., Ropelewski, C., Wang, J., Jenne, R., and Joseph, D.: The NCEP/NCAR 40-year reanalysis project, B. Am. Meteorol. Soc., 77, 437-471, doi:10.1175/15200477(1996)077;0437:TNYRP;2.0.CO;2, 1996.

Kestin, T. S., Karoly, D. J., Yano, J.-I., and Rayner, N. A.: Timefrequency variability of ENSO and stochastic simulations, J. Climate, 11, 2258-2272, 1998.

Kim, K.-Y., Park, R. J., Kim, K.-R., and Na, H.: Weekend effect: anthropogenic or natural?, Geophys. Res. Lett., 37, L09808, doi:10.1029/2010GL043233, 2010. 
Kolmogorov, A. N.: Dokl. Akad. Nauk., 26, 115, 1941, (reprinted in: Proc. R. Soc. Lond. A, 434, 9-13, 1991).

Lau, K.-M., and Weng, H.-Y: Climate signal detection using wavelet transform: How to make a time series sing, B. Amer. Meteor. Soc., 76, 2391-2402, 1995.

Lilly, D. K.: Stratified turbulence and the mesoscale variability of the atmosphere, J. Atmos. Sci., 40, 749-761, 1983.

Lilly, D. K.: Two-dimensional turbulence generated by energy sources at two scales, J. Atmos. Sci., 46, 2026-2030, 1989.

Lin, C.-Y., Liu, S. C., Chou, C. C.-K., Liu, T. H., Lee, C.-T., Yuan, C.-S., Shiu, C.-J., and Young, C.-Y.: Long-range transport of Asian dust and air pollutants to Taiwan, Terr. Atmos. Ocean. Sci., 15(5), 759-784, 2004.

Lin, C.-Y., Liu, S. C., Chou, C. C.-K., Huang, S.-J., Liu, C.-M., Kuo, C.-H., and Young, C.-Y.: Long-range transport of aerosols and their impact on the air quality of Taiwan, Atmos. Environ., 39, 6066-6076, 2005.

Lindborg, E.: Can the atmospheric kinetic energy spectrum be explained by two-dimensional turbulence?, J. Fluid Mech., 388, 259-288, 1999.

Madden, R. A. and Julian, P. R.: Observations of the 40-50-day tropical oscillation - A review, Mon. Weather. Rev., 122, 814837, 1994

Mallat, S. G.: A Wavelet Tour of Signal Processing. 2nd. Ed., Academic Press, 637 pp., 1999.

Mao, H. and Talbot, R.: $\mathrm{O}_{3}$ and $\mathrm{CO}$ in New England: Temporal variations and relationships, J. Geophys. Res., 109, D21304, doi:10.1029/2004JD004913, 2004.

Meyers, S. D., Kelly, B. G., and O'Brien, J. J.: An introduction to wavelet analysis in oceanography and meteorology: With application to the dispersion of Yanai waves, Mon. Weather Rev., 121, 2858-2866, 1993.

Miller, M. J. and Smolarkiewicz, P. K. (Eds.): Predicting weather, climate and extreme events, J. Comput. Phys., 227(7), 34293730, 2008.

Morlet, J., Arens, G., Fourgeau, I. and Giard, D.: Wave propagation and sampling theory, Geophysics, 47, 203-236, 1982.
Nastrom, G. D. and Gage, K. S.: A climatology of aircraft wavenumber spectra observed by commercial aircraft, J. Atmos. Sci., 42, 950-960, 1985.

Nastrom, G. D., Gage, K. S., and Jasperson, W. H.: Atmospheric kinetic energy spectrum, $10^{0}-10^{4} \mathrm{~km}$, Nature, 310, 36-38, 1984.

Peng, C.-K., Buldyrev, S. V., Goldberger, A. L., Havlin, S., Sciortino, F., Simons, M., and Stanley, H. E.: Long-range correlations in nucleotide sequences, Nature, 356, 168-170, 1992.

Peng, C.-K., Buldyrev, S. V., Goldberger, A. L., Havlin, S., Simons, M., and Stanley, H. E.: Finite-size effects on long-range correlations: Implications for analyzing DNA sequences, Phys. Rev. E., 47, 3730-3733, 1993.

Peng, C.-K., Buldyrev, S. V., Havlin, S., Simons, M., Stanley, H. E., and Goldberger, A. L.: Mosaic organization of DNA nucleotides, Phys. Rev. E., 49, 1685-1689, 1994.

Peng, C.-K., Havlin, S., Stanley H. E., and Goldberger, A. L.: Quantification of scaling exponents and crossover phenomena in nonstationary heartbeat time series, Chaos, 5, 82-87, 1995.

Seinfeld, J. H. and Pandis, S. N.: Atmospheric Chemistry and Physics: From Air Pollution to Climate Change, 2nd. ed., 1288 pp., 2006.

Stephens, S., Madronich, S., Wu, F., Olson, J. B., Ramos, R., Retama, A., and Muñoz, R.: Weekly patterns of México City's surface concentrations of $\mathrm{CO}, \mathrm{NO}_{\mathrm{x}}, \mathrm{PM}_{10}$ and $\mathrm{O}_{3}$ during 19862007, Atmos. Chem. Phys., 8, 5313-5325, doi:10.5194/acp-85313-2008, 2008.

Talbot, R., Mao, H., and Sive, B.: Diurnal characteristics of surface level $\mathrm{O}_{3}$ and other important trace gases in New England, J. Geophys. Res., 110, D09307, doi:10.1029/2004JD005449, 2005.

Torrence, C. and Compo, G. P.: A practical guide to wavelet analysis, B. Am. Meteorol. Soc., 79, 61-78, 1998.

Tuck, A.: Atmospheric Turbulence: A Molecular Dynamics Perspective, Oxford University Press, 157 pp., 2008.

Wilks, D. S.: Statistical Methods in the Atmospheric Sciences, Academic Press, 467 pp., 1995.

Wornell, G.: Signal Processing with Fractals: A Wavelet-Based Approach, Prentice Hall, 177 pp., 1996. 